\title{
Mutated SASH1 promotes Mitf expression in a heterozygous mutated SASH1 knock-in mouse model
}

\author{
ZEXI XU ${ }^{1,2^{*}}$, YADONG $\mathrm{LI}^{3 *}$, DAHONG WANG ${ }^{1 *}$, DAOQIU WU ${ }^{4 *}$, JINYUN WANG $^{5}$, \\ ${\text { LIAN } \text { CHEN }^{3} \text {, YINQIAN DENG }}^{3}$, JING ZHANG ${ }^{1}$, ZHIXIONG WU ${ }^{3}$, XIN WAN ${ }^{3}$, QIANFAN LIU ${ }^{3}$, \\ HAI HUANG ${ }^{4}$, PINGSHENG HU ${ }^{1}$, JIAWEI ZENG ${ }^{6}$ and DING'AN ZHOU ${ }^{1,4}$ \\ ${ }^{1}$ Clinical Research Center, The Affiliated Hospital of Guizhou Medical University, Guiyang, Guizhou 550004; \\ ${ }^{2}$ Department of Oncology Center, Renmin Hospital of Wuhan University, Wuhan, Hubei 430060; \\ ${ }^{3}$ Clinical College and ${ }^{4}$ School of Clinical Laboratory Sciences, Guizhou Medical University, Guiyang, Guizhou 550004; \\ ${ }^{5}$ School of Biology and Engineering, Guizhou Medical University, Guiyang, Guizhou 550025; \\ ${ }^{6}$ Department of Clinical Laboratory, Mianyang Central Hospital, Mianyang, Sichuan 621000, P.R. China
}

Received March 12, 2020; Accepted June 3, 2020

DOI: $10.3892 /$ ijmm.2020.4652

\begin{abstract}
The SAM and SH3 domain-containing 1 (SASH1) genes have been identified as the causal genes of dyschromatosis universalis hereditaria (DUH); these genes cause the pathological phenotypes of DUH, and $S A S H 1$ variants have been shown to regulate the abnormal pigmentation phenotype in human skin in various genodermatoses. However, investigations into the mutated $S A S H 1$ gene have been limited to in vitro studies. In the present study, to recapitulate the molecular pathological phenotypes of individuals with DUH induced by $S A S H 1$ mutations, a heterozygous BALB/c mouse model, in which the human SASH1 c.1654 T>G (p. Tyr 551Asp, Y551D) mutation was knocked in was first generated. The in vivo functional experiments on Y551D SASH1 indicated that the increased expression of microphthalmia-associated transcription factor (Mitf) was uniformly induced in the tails of heterozygous BALB/c mice, and an increased quantity of Mitf-positive epithelial cells was also detected. An increased expression of Mitf- and Mitf-positive cells was also demonstrated in the epithelial tissues of Y551D-SASH1 affected individuals. In the present study, Mitf expression was
\end{abstract}

Correspondence to: Professor Ding'an Zhou, Clinical Research Center, The Affiliated Hospital of Guizhou Medical University, 28 Guiyi Street, Yunyan, Guiyang, Guizhou 550004, P.R. China E-mail: 460318918@qq.com; 081023094@fudan.edu.cn

Professor Jiawei Zeng, Department of Clinical Laboratory, Mianyang Central Hospital, 12 Changjia Lane, Jingzhong Street, Mianyang, Sichuan 621000, P.R. China

E-mail: 1075650644@qq.com; zjweee@sina.cn

${ }^{*}$ Contributed equally

Key words: Y551D SASH1, mouse model, microphthalmia-associated transcription factor, dyschromatosis universalis hereditaria also found to be increased by Y551D SASH1 in vitro. Taken together, these findings indicate that the upregulation of Mitf is the bona fide effector of the Y551D SASH1-mediated melanogenesis signaling pathway in vivo. SASH1 may function as a scaffold molecule for the assembly of a SASH1-Mitf molecular complex to regulate Mitf expression in the cell nucleus and thus to promote the hyperpigmented phenotype in the pathogenesis of DUH and other genodermatoses related to pigment abnormalities.

\section{Introduction}

Dyschromatosis universalis hereditaria (DUH) is a clinically heterogeneous disorder that is characterized by evident mottled pigmentation over the entire body of affected individuals. In 2003, similar Chinese DUH pedigrees were discovered with dyschromatosis symmetrica hereditaria (DSH) with autosomal dominant DUH (1) and these cases were subsequently diagnosed as DUH rather than DSH. In the $10.2 \mathrm{Mb}$ region on chromosome 6 (6q24.2-q25.2), a c.1654 $\mathrm{T}>\mathrm{G}$ (p. Tyr 551Asp, Y551D) mutation in exon 14 of SAM and SH3 domain-containing 1 (SASH1), a c.1547 T>C (p. Leu 515 Pro, L515p) mutation in exon 13, and a c.1528 G>A (p. Glu 509 Lys, E509K) mutation in exon 13 were previously identified in 3 pedigrees $(2,3)$. Further in vitro analyses indicated that the enhanced expression of melanogenic-related partners was induced by SASH1 mutations, and mosaic-like phenotypes of SASH1, melanin and melanogenic enzymes were detected in the epithelial tissues from the lesional areas of DUH-affected individuals (2-4). Recently, novel SASH1 mutations [c.1784T >C (p. M595T) and c.1651T >C (p. Y551H)] were found to be associated with Chinese families with DUH (5). A c.1556 G->A (p. S519N) heterozygous mutation in exon 13 of the SASH1 gene was reported in familial lentigines (6). A c.1519T $>\mathrm{G}$ (p.Ser507Ala.) heterozygous transition mutation in exon 13 of the SASH1 gene was also identified in a Chinese family with multiple lentigines (7). Two novel mutations, c.1537A $>C$ (p. Ser513Arg) and 1527_1530dupAAGT (p. Leu511Lysfs*21) in 
the SASHI gene, were identified in 3 pediatric patients with lentiginous phenotypes, and the clinical presentations revealed that SASH1-related phenotypes can exhibit hyper- and hypopigmentation on the trunk and extremities (8). A homozygous missense substitution (c.1849G>A; p. Glu617Lys) in the $S A S H 1$ gene was identified to be associated with genodermatosis in an autosomal recessive manner (9). These studies indicate that SASH1 has gradually become an important gene that mediates melanin production in the process of human skin pigmentation in various genodermatoses related to pigment abnormalities. SASH1 variants may cause autosomal-dominant or autosomal-recessive genodermatosis (10).

However, the SASH1-mediated melanogenesis-molecular signaling networks that were found in vitro and the investigations on $\mathrm{SASH} 1$ gene functions reported by other dermatologists are limited to in vitro evaluations. Further investigations are required to verify the SASH1-involved signaling networks and/or the $S A S H 1$ variant functions in mammals.

As the gene roles of Y551D SASH1 in the induction of a hyperpigmentation phenotype, which were identified in vitro, are more significant than those of E509K and L515P SASH1, heterozygous and homozygous mouse models with a c.1654 $\mathrm{T}>\mathrm{G}$ mutation of human SASH1 were established in the present study. The mouse models with a c.1654 $\mathrm{T}>\mathrm{G}$ mutation of SASH1 recapitulated some molecular pathological phenotypes in the epithelial tissues of the tails of heterozygous mice; these phenotypes were similar to those in the skin epithelial tissues from the lesional areas of DUH-affected individuals and support the results of the in vitro cell function experiments that have been described previously (2-4).

\section{Materials and methods}

In vitro transcription of Cas $9 \mathrm{mRNA}$ and $s g R N A$ and construction of donor vector. The sgRNA was designed and transcribed in vitro. The sequence of the sgRNA was as follows: 5'-CGCGGCCATGGAGGAGGACG-3' (CGG) (PAM shown in brackets). The Cas9 expression construct was linearized, transcribed in vitro and extracted with phenol-chloroform. The donor vector [XM709442 Sash1-h SASH1(Y551D) DONOR] was designed and constructed. The detailed mSash1-hSASH1(Y551D) Cas9-KI Targeted Genomic Sequence is illustrated in Data S1. sgRNAs directed Cas9 endonuclease cleavage at exon 1 near the start codon ATG to create a double-stranded break (DSB). Such breaks were repaired, leading to the insertion of hSASH1 (Y551D)-PolyA after the start codon, by homologous recombination. The hSASH1 (Y551D)-PolyA cassette was placed after the translational start codon ATG of the mouse Sashl gene. The strategy of generating the mSash-hSASH1(Y551D) gene knock-in BABL/cJ mice is shown in Fig. 1A. The structure map of the XM709442 Sash1-hSASH1 (Y551D) donor vector is illustrated in Fig. 1B.

Animals and construction of Sash-hSASH1(Y551D) gene knock-in $B A B L / c J$ mice. All animal experiments were conducted according to experimental practices and standards approved by the Ethics Committee of the Nanjing Biomedical Institute of Nanjing University and Guizhou Medical University (License no. 1800125). The N-terminus of the mouse Sash1 gene (Gene ID: 70097) locus was the knock-in site, and the knock-in fragment of hSASH1 (Y551D)-PolyA (human SASH1 Gene ID: 23328) was inserted at the ATG start codon in exon 1 of murine Sash1. Therefore, human SASH1 and EGFP were expressed under the control of the endogenous mouse SASH1 promoter/enhancer elements. Cas9 mRNA, sgRNAs and donor vector were co-injected into zygotes or fertilized eggs of a total of $100 \mathrm{BALB} / \mathrm{cJ}$ mice (Nanjing Biomedical Research Institute of Nanjing University) by microinjection. Among 54 newborns containing hSASH1(Y551D)-PolyA cassettes on double DNA chains, 6 mice, including 3 male BALB/cJ mice [animal strain: (T004567) BALB/cNju-h SASH11 em1Cin(Y551D)/Nju ] and 3 female mice [animal strain: (T004567) BALB/cNju-h SASH11 em1Cin(Y551D)/Nju], were designated as the F0 generation mice, and their genotypes were confirmed by PCR and DNA sequencing. The genotyping results of Southern blot analysis among 8 mice are showed in Table I. These F0 generation mice were housed and maintained under specific pathogen-free (SPF) conditions at the Experimental Animal Center of Nanjing Biomedical Research Institute of Nanjing University. The environment temperature was maintained at $22-28^{\circ} \mathrm{C}$; the humidity was 40-60\%; the noise was lower than $60 \mathrm{~dB}$, the ammonia concentration was not $>20 \mathrm{ppm}$, and the number of air changes shall was $10-20$ times/h in the SPF experimental animal center. The sterile full nutrition granule material sterilized by 60 Co gamma-ray was used to feed the adult mice with doses of 3-7 g/day or the young mice with 1-3 g/day. A small amount of sunflower seeds were fed to the mice regularly and autoclave purified water after bottling with change frequency of 2-3 times per week was provided for drinking.

Mouse genotyping. All procedures were approved by the Institutional Animal Care and Use Committee at Guizhou Medical University and were consistent with the Guide for the Care and Use of Laboratory Animals published by the National Institutes of Health. F1 generation mice were produced by mating F0 generation mice with a wild-type BALB/c mouse (Nanjing Biomedical Research Institute of Nanjing University), and F2 generation mice were produced by mating F1 generation mice with a wild-type BALB/c mouse and F3 generation mice were produced by in proper order. All bred mice of the $\mathrm{F} 2$ and $\mathrm{F} 3$ generations at the age of 2 to 4 weeks were exposed to $60 \% \mathrm{O}_{2}$ plus $2 \%$ isoflurane with a $0.5 \mathrm{l} / \mathrm{min}$ isoflurane flow and a $0.5 \mathrm{l} / \mathrm{min}$ oxygen flow in a mobile anesthesia system for small animals (R510-46, RWD Life Science Co. Ltd.). The lower doses (the dose of isoflurane was 1-2\%) and higher doses (the dose of isoflurane was 4-5\%) of isoflurane were used to demonstrate the effects of the non-anesthetic vs. the anesthetic dose of isoflurane on the genotoxic effects of hyperoxia. The detailed procedure has been previously described in the study by Kundumani-Sridharan et al (11). Following the anesthesia coma i.e., the limbs of the anesthetized mice did actively contract when needling the syringe needle, the anesthetized mice were euthanized by cervical dislocation and the tail biopsies of the mice were cut off and collected for further analyses. The remaining bodies of the mice were collected in special mouse body bags for centralized processing, which were prepared by the specific pathogen-free Experimental Animal Center of Clinical Research Center, the Affiliated Hospital of Guizhou Medical University. The EasyPure Genomic DNA 


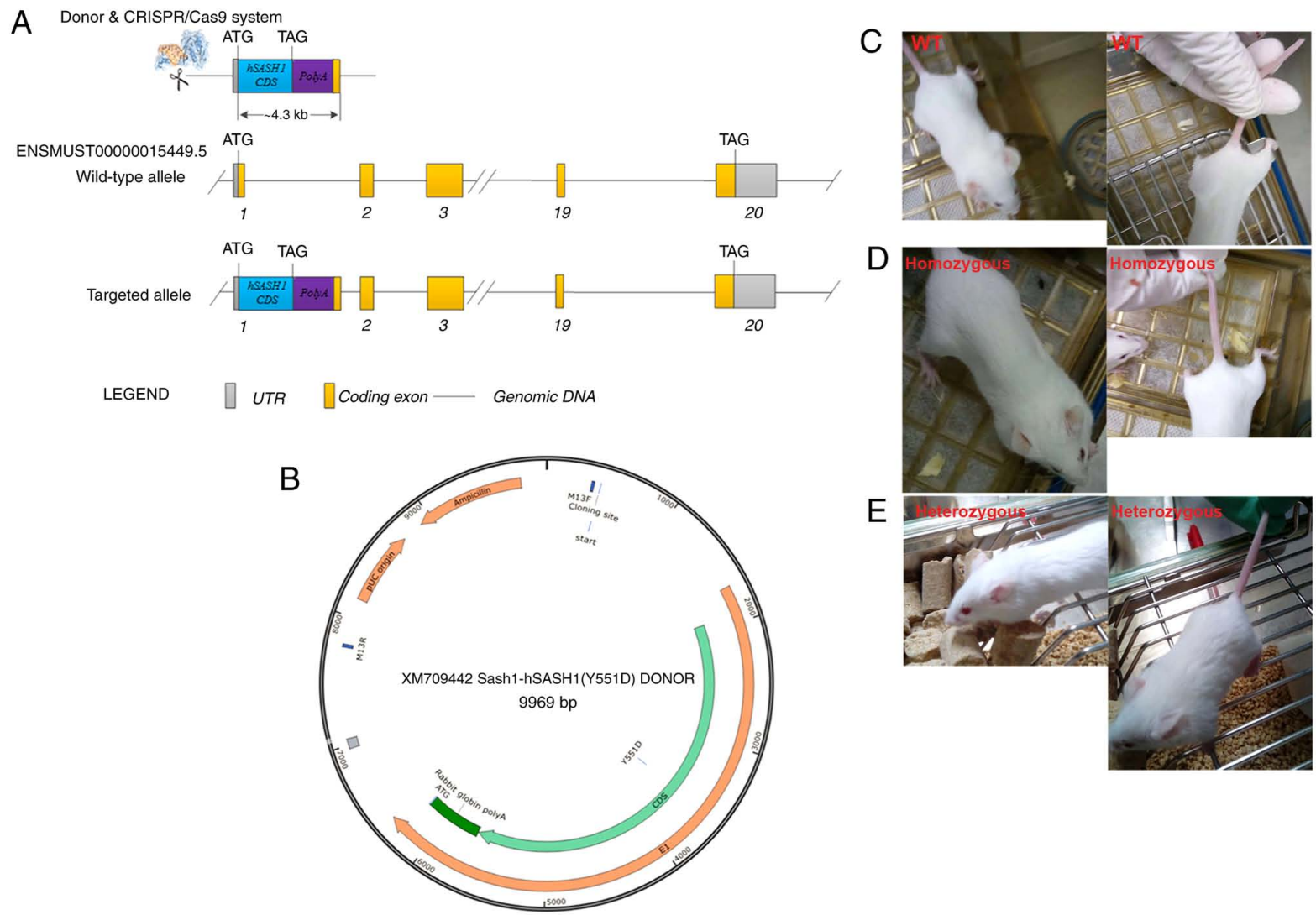

Figure 1. Construction of mSash-hSASH1(Y551D) gene knock-in BABL/cJ mice. (A) Strategy for the construction of mSash-hSASH1(Y551D) gene knock-in BABL/cJ mice. (B) Schematic diagrams of the structure of the donor vector [XM709442 Sash1 hSASH1(Y551D) DONOR]. The donor vector contained $9969 \mathrm{bp}$ of nucleotides, and the detailed sequences of the donor vector are indicated in the Sash1-hSASH1(Y551D) Cas9-KI Targeted Genomic Sequence (Data S1). (C-E) No obvious hyperpigmented spots were observed in the mouse tails. Representative images of wild-type, homozygous and heterozygous mice in the F2 generation are shown. WT, wild-type.

kit (cat. no. EE101-01, TransGen Biotech) was used to extract tissue DNA from the mouse tails as described in the protocol provided by the manufacturer.

The F1 generation pups of the Sash-hSASH1 (Y551D) gene knock-in BABL/cJ mice were genotyped by PCR, followed by sequencing and Southern blot analysis. The analyses of genotyping, sequencing and Southern blot analysis of F1 generation mice were performed by the Nanjing Biomedical Research Institute of Nanjing University, and the detailed protocols of these analyses were not provided by the Nanjing Biomedical Research Institute. The identification strategy is presented in Fig. S1A. The primer sequences for identification and sequencing are presented in Tables SI and SII. The F2 and F3 generation pups of the mSash-hSASH1 (Y551D) gene knock-in BABL/cJ mice were identified by PCR that amplified the mutated knock-in bands of the human SASHI gene and the wild-type bands of the mouse Sashl gene. The PCR primer sequences and detailed information regarding the size of the amplified bands, as well as an illustration of how the genotype of the transgenic mice was determined according to the amplified bands, are demonstrated in Table SIII.

Reverse transcription PCR (RT-PCR). Total RNA was extracted from the mouse tails using the AxyPrep Multisource Total RNA Miniprep kit 50-prep (AP-MN-MS-RNA-50, Corning Axgene) according to the protocol provided by the manufacturer. Total RNA extracted from the mouse tails was reverse transcribed into cDNA using the Easy Script One-Step gDNA Removal and cDNA Synthesis Super Mix (AE311-02, Transgene), and this cDNA was used as template for RT-PCR. PCR was performed with 2X Taq Plus PCR MaterMix (KT205-01, Tiangen Biotech Co., Ltd.) was used to amplify the cDNA and followed by nucleic acid electrophoresis to evaluate SASH1 or Sash1 expression in the tail tissues of F2 and $\mathrm{F} 3$ generation. The thermocycling conditions were as follows: $94^{\circ} \mathrm{C}, 5 \mathrm{~min} ; 94^{\circ} \mathrm{C}, 30 \mathrm{sec}, 60^{\circ} \mathrm{C}, 30 \mathrm{sec}, 72^{\circ} \mathrm{C}, 30 \mathrm{sec}$, 30 cycles; $72^{\circ} \mathrm{C}, 5 \mathrm{~min}, 4^{\circ} \mathrm{C}$ continuously. The $S A S H 1$ gene exhibits high conservation among different species, and the human SASH1 gene exhibits a high similarity with the mouse Sash1 gene in the whole cDNA sequence. The fragment containing $155 \mathrm{bp}$ from nucleotide 1954 to nucleotide 2108, is located in the SASH1 SAM1 domain, was amplified to analyze its expression in wild-type, homozygous and heterozygous mice of the F2 and F3 generations. The following primers were used to amplify $S A S H 1$ and $G A P D H$ from the mouse tails, and the sequences of the SASH1 and GAPDH primers were as follows: SASH1 forward, 5'-CCCACTTTCCTGTTC AATG-3' and reverse, 5'-TGGTCGCTGTTACTGTCATAC-3'; and GAPDH forward, 5'-CACCCACTCCTCCACCTTTG-3' and reverse, 5'-ACCACCCTGTTGCTGTAGCC-3'.

Western blot analysis and IP-WB. A total of 11 heterozygous mice of the F2 generation and 10 heterozygous mice of the F3 generation, which harbored the mouse Sash1-human 
Table I. Genotyping records of the F0 generation mice.

\begin{tabular}{lll}
\hline Mouse ID no. & Sex & Southern blot analysis results \\
\hline 3 & Male & Negative \\
5 & Male & Positive \\
8 & Male & Negative \\
25 & Male & Positive \\
56 & Male & Positive \\
17 & Female & Positive \\
35 & Female & Positive \\
54 & Female & Positive \\
\hline
\end{tabular}

SASH1(Y551D)-PolyA cassette in one DNA chain and mouse Sash1 in the other DNA chain, 2 homozygous mice of the F2 generation and 1 homozygous mouse of the F3 generation, which both harbored the mouse Sash1-human SASH1 (Y551D)-PolyA cassette in both DNA chains, and 3 wild-type mice of the F2 generation, and 4 heterozygous mice of the F3 generation, which both contained the mouse Sash1 gene in both DNA chains, were identified according to the mouse genotyping results.

To identify the expression of exogenous human SASH1 in the heterozygous mice and homozygous mice, and to determine the effects of exogenous human SASH1 on melanogenic enzymes or melanogenic partners, mouse tail tissues from mice of the F2 and F3 generations were sheared by ultrasonic breaking, lysed with RIPA lysis buffer and subjected to western blot analysis. The protein concentration was quantified by BCA methods, $35 \mu \mathrm{g}$ protein per lane was loaded and followed by electrophoresis using $5 \%$ concentration gel and $10 \%$ of separation gel. After electrophoresis, the protein on the separation gel was transferred to polyvinylidene difluoride membrane and blocked with $10 \%$ fat-free milk for $1 \mathrm{~h}$ at room temperature. The primary antibodies was diluted with primary antibody dilution buffer (P0023A, Beyotime Biotechnology) and was incubated at $4^{\circ} \mathrm{C}$ for one night. The enhanced chemiluminescent (ECL) Reagent (cat. no. KF005, Affinity Biosciences) was used to visualize the protein bands. The densitometry of protein bands was quantified by Quantity One software (Vision 4.6.2, the Discovery Series) and analyzed by SPSS16.0 software (IBM, International Business Machine).

The primary antibodies used for western blot analysis were rabbit anti-SASH1 polyclonal antibody (1:500 dilution, cat. no. NBP-26650, Novus Biological, USA), rabbit anti-microphthalmia-associated transcription factor (Mitf; C5) monoclonal antibody (1:500 dilution, cat. no. NB110-10872, Novus Biologicals, LLC) or rabbit anti-Mitf polyclonal antibody (1:500 dilution, cat. no. ab20663, Abcam), rabbit anti-GNAS polyclonal antibody (1:500 dilution, cat. no. C2C3-2, GeneTex) and rabbit anti-phospho-ERK1/2 (Thr202/Tyr204) monoclonal antibody (1:1,000 dilution, cat. no. 9101, Cell Signal Technology, Inc.), anti-p44/42 MAPK (Erk1/2) (137F5) rabbit mAb (1:1,000 dilution, cat. no. 4695, Cell Signal Technology, Inc.), GFP mouse monoclonal antibody (1:2,000 dilution, cat. no. T0005, Affinity Biosciences), mouse anti- $\beta$-tubulin mouse monoclonal antibody 1:2,000 dilution, (cat. no. 66240-1-Ig, Proteintech Group, Inc.) and rabbit anti-GAPDH polyclonal antibody (1:2,000 dilution, cat. no. GTX100118, GeneTex). The secondary antibodies used [peroxidase-conjugated AffiniPure Goat Anti-Rabbit IgG $(\mathrm{H}+\mathrm{L})$ or peroxidase-conjugated AffiniPure Goat Anti-Mouse IgG (H+L) (cat. no. 111-035-003, Jackson ImmunoResearch Laboratories, Inc.] were diluted 1:10,000 and incubated with the membranes at room temperature for $1 \mathrm{~h}$.

The pEGFP-C3-SASH1 vector was introduced into 293T cells (the Cell Bank of Chinese Academy of Sciencesa). The transfected 293T cells were lysed with IP-WB cell lysis buffer (P0013J, Beyotime Biotechnology). GFP-SASH1 was immunoprecipitated with an anti-GFP antibody (1:100 dilution, cat. no. T0005, Affinity Biosciences). The immunoprecipitates were captured by Protein A/G plus-agarose (sc-2003, Santa Cruz Biotechnology, Inc.), and the associated endogenous Mitf (cat. no. ab20663, Abcam) was analyzed.

Immunohistochemical analyses. After acquiring the consents of the affected individuals and the normal controls, the skin epithelial tissues from the hyperpigmented lesional areas of a 28-year-old female Y551D-affected individual and the normal skin epithelial tissues from 2 normal controls were obtained at the Affiliated Hospital of Guizhou Medical University on January 20, 2020. The document of patient consent had been signed by the affected individual and 2 normal controls, and ethics approval was provided by the Ethics Committee of the Affiliated Hospital of Guizhou Medical University. The epithelial tissues from the tail biopsies of the mice of the F2 and F3 generations, and the skin epithelial tissues from hyperpigmented lesional areas of Y551D-affected individuals and normal controls were fixed in $10 \%$ formalin at $4^{\circ} \mathrm{C}$ for $24 \mathrm{~h}$ and then embedded in paraffin. Paraffin sections $(5-\mu \mathrm{m}$-thick) were incubated at $60^{\circ} \mathrm{C}$ for $3 \mathrm{~h}$ and then deparaffinized and rehydrated using xylene and an ethanol gradient. The sections were then incubated with the rabbit anti-Mitf (C5) monoclonal antibody (1:200 dilution, cat. no. NB110-10872, Novus Biologicals, LLC), rabbit anti-SASH1 polyclonal antibody (1:200 dilution, cat. no. NBP-26650, Novus Biologicals, LLC), rabbit anti-Mitf (C5) monoclonal antibody (1:200 dilution, cat. no. NB110-10872, Novus Biologicals, LLC) at $37^{\circ} \mathrm{C}$ for $1 \mathrm{~h}$ and then at $4^{\circ} \mathrm{C}$ for at least $8 \mathrm{~h}$. After washing with PBS 3 times, the sections were incubated with the horseradish peroxidase-linked anti-rabbit and anti-mouse universal secondary antibodies provided by the Immunochromogenic Kit (KIT-5006, MXB Biotechnologies) for $1 \mathrm{~h}$ at $37^{\circ} \mathrm{C}$. Finally, the sections were counterstained with hematoxylin staining solution (CTS-1090, MXB Biotechnologies at $37^{\circ} \mathrm{C}$ for $20 \mathrm{~min}$, and photographed under a positive position microscope BX51 (Olympus Corp.). The staining intensity and percentage of positive cells in the sections of the 3 groups of mice, including wild-type mice, homozygous mice and heterozygous mice of the F2 generation and F3 generation, were calculated and scored as previously described (4). The Mitf-positive cells in the epithelial tissues of tail biopsies from mice of the F2 and F3 generations and the Mitf-positive cells in the affected epithelial layers from skin lesional areas of Y551D-affected patients were examined. 
A
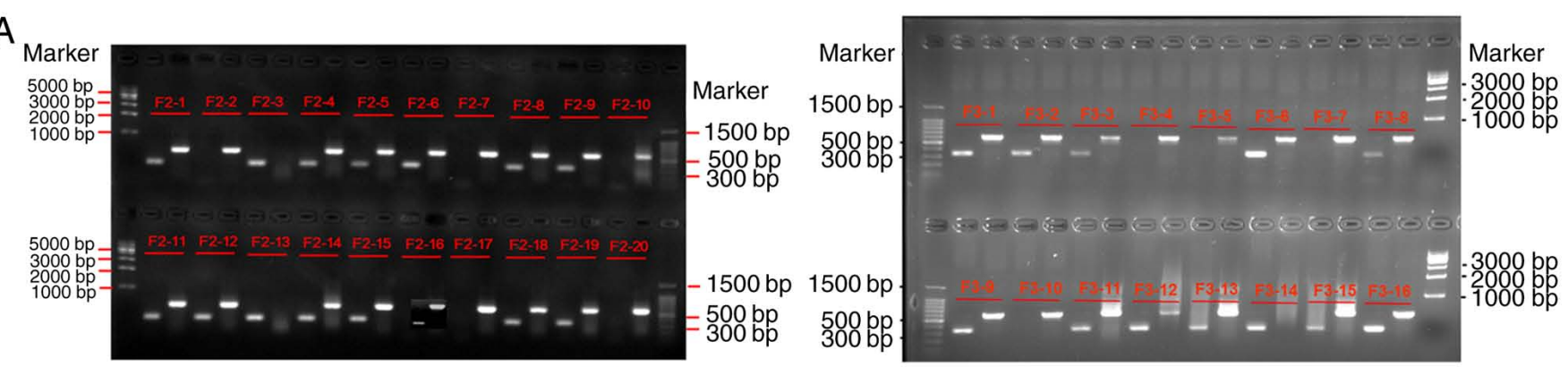

B

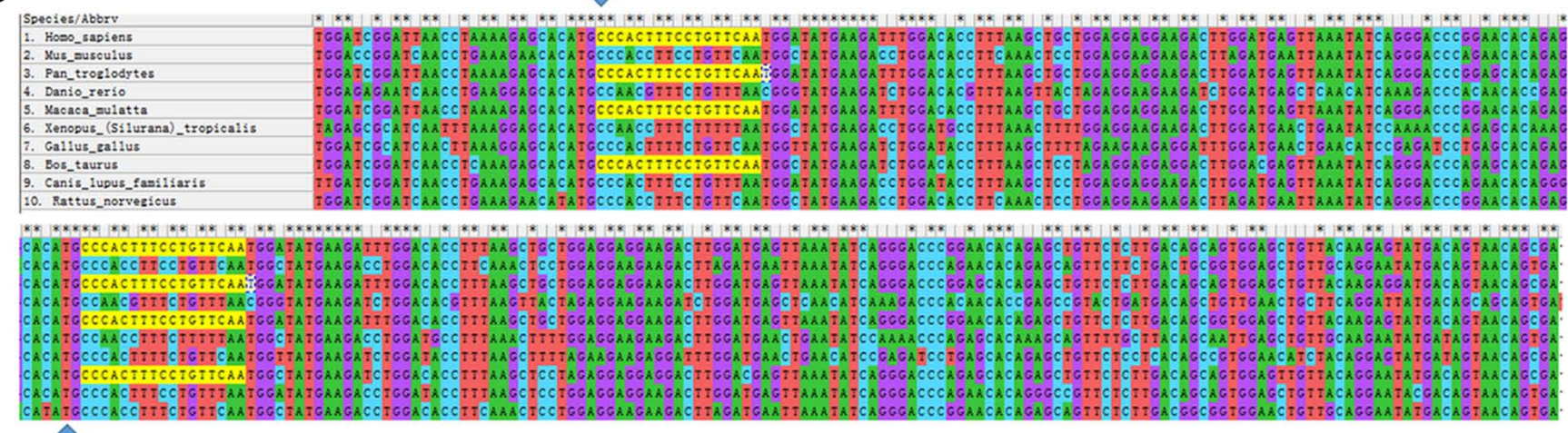

C
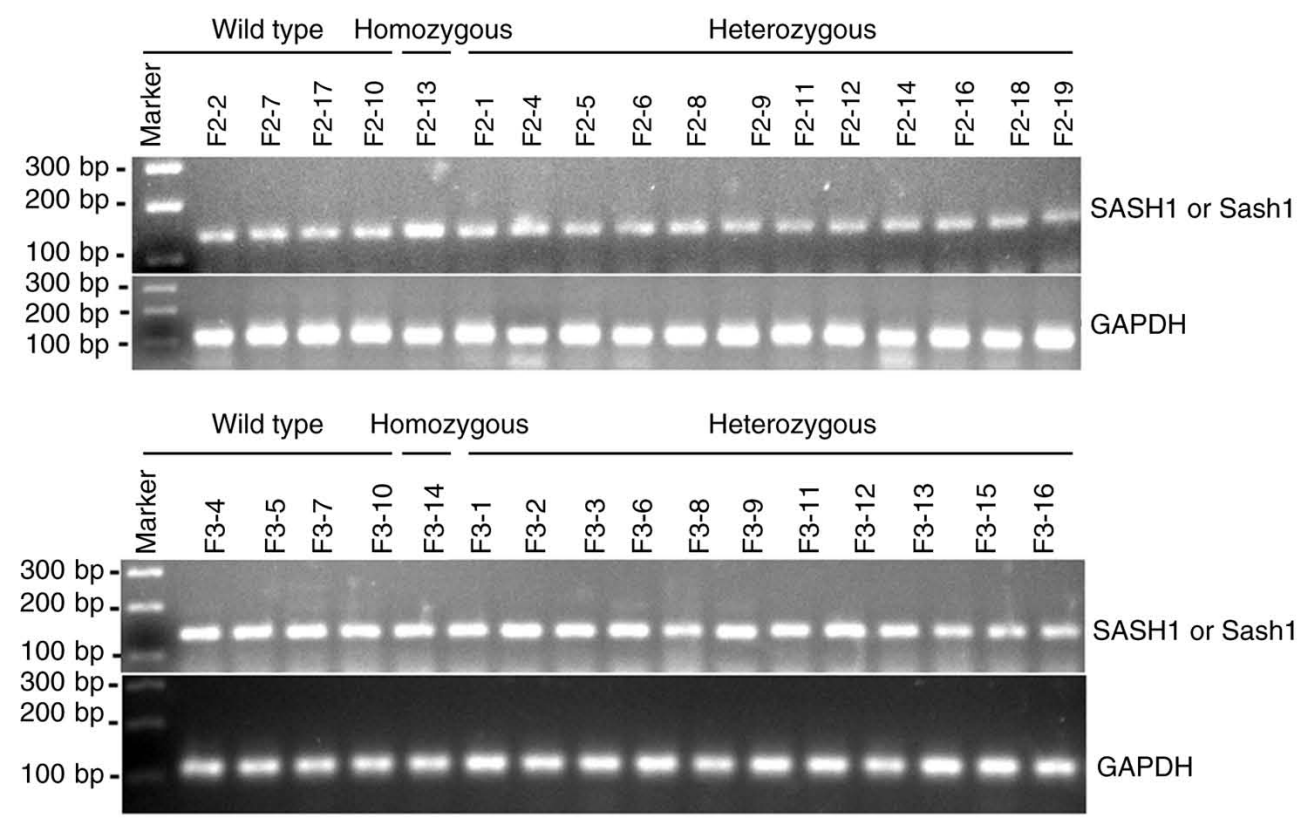

Figure 2. Mouse genotyping results and the expression of the human SASH1 gene and/or mouse Sash1 gene in the F2 generation and F3 generation mice. (A) Tail biopsies of the mice of the F2 and F3 generations were collected, sheared and trypsinized at $56^{\circ} \mathrm{C}$. Tissue DNA from the mouse tails was extracted, and the genotyping of the F2 and F3 generation pups of mSash-hSASH1(Y551D) gene knock-in BABL/cJ mice was performed by PCR using primers (shown in Table SIII) to amplify the mutated knock-in bands of the human SASH1 gene and the wild-type bands of the mouse Sash1 gene. Pups of F2-2, F2-7 and F2-17 of the F2 generation and those of F3-4, F3-5, F3-7 and F3-10 of the F3 generation mice were identified as wild-type. Pups of F2-3 and F2-13 of the F2 generation and F3-14 of the F3 generation were identified to be homozygous. Other pups of the F2 and F3 generations were heterozygous. (B) The conservation of the SASH1 gene in different species was analyzed by MEGA-X software, and high conservation of the SASHI gene was shown in most species. The blue arrows indicate the segments of human SASH1 gene and/or mouse Sash1 gene which were amplified by RT-PCR. (C) The expression of the human SASH1 or mouse Sash1 gene in wild-type, homozygous and heterozygous mice of the F2 and F3 generations was assessed by RT-PCR. RT-PCR demonstrated that at the transcriptional level, the human $S A S H 1$ and mouse Sashl genes are expressed in wild-type, homozygous and heterozygous mice.

Melanin staining. Melanin staining in the skin epithelial tissues of the normal control and Y551D SASH1-affected individuals was performed as previously described (3).

Statistical analysis. The protein densitometry values of western blot analysis were first measured using Quantity One software (version 4.6.2) and analyzed using the homogeneity of variance test, followed by a one-factor analysis of variance (ANOVA) with LSD correction or Tukey's test in SPSS version 16.0 to generate the required P-values. The data are presented as the means \pm standard error of the mean (SEM). The total scores and positively stained cells of each visual 
field of the immunohistochemistry sections were scored and analyzed using the homogeneity of variance test and a one-factor analysis of variance (ANOVA) with LSD correction or Tukey's test in SPSS version 16.0 to generate the required P-values. The cartograms were made and plotted using GraphPad Prism 5 (GraphPad Software, Inc.).

\section{Results}

The human SASH1 gene and/or the mouse Sashl gene are both expressed in hSASH1(Y551D) gene knock-in BABL/cJ mice of the F2 and F3 generations. A total of 6 mice (3 males and 3 females) of the F0 generation were ultimately identified as positive Sash-hSASH1(Y551D) gene knock-in BABL/cJ mice by PCR and sequencing. A total of $11.1 \%$ (6/54) of the live pups obtained presented a mutational event. Mice nos. 64, 66, 68, 72, 75 and 78 of the F1 generation were identified to be positive by PCR amplification and point mutation sequencing. The identification strategy is shown in Fig. S1A. The Sequencing results indicated that the human SASH1 (c.1654 T>G mutation)-PolyA cassette was successfully inserted into the mouse genome (Fig. S1B). The results of Southern blot analysis and point mutation sequencing are presented in Fig. S1C-E. Regretfully, in both the homozygous and heterozygous mice, no hyperpigmentation spots or hypopigmentation spots were found within the tails or other areas of the mouse bodies (Fig. 1C, D and E).

The genotypes of 16 mice of the $\mathrm{F} 2$ generation and 16 mice of the F3 generation were further identified by PCR. Nucleic acid electrophoresis indicated that of the F2 generation mice, 3 mice were wild-type mice, 2 were homozygous and 11 were heterozygous (Fig. 2A). Among the F3 generation mice tested, 4 mice were wild-type mice, 1 was homozygous, and 11 were heterozygous (Fig. 2A). The genotyping results are presented in Table II. The expression of the human SASHI gene and/or mouse Sashl gene was further analyzed in the wild-type, homozygous and heterozygous mice of the F2 and F3 generations. The conservation of the $S A S H 1$ gene in different species was also assessed, and a high degree of conservation between mice and humans was demonstrated (Fig. 2B). Therefore, the expression of the human SASHl gene and mouse Sashl gene at the transcriptional level was analyzed by RT-PCR using the same pair of primers.

The results of RT-PCR indicated that the human SASHI gene was expressed in the homozygous and heterozygous mice, but also in the $S A S H 1$ gene wild-type and heterozygous mice (Fig. 2C). Therefore, the human SASHI gene and/or mouse Sashl gene were both expressed in the wild-type, homozygous and heterozygous mice of the F2 and F3 generations.

GNAS is not uniformly induced in heterozygous hSASH1(Y551D) gene knock-in BABL/cJ mice. Upon ligand binding to $G$ protein-coupled receptors (GPCRs), GPCRs impart a signal to heterotrimeric $G$ proteins, which are composed of $\alpha$-, $\beta$ - and $\gamma$-subunits, resulting in the detachment of the $\alpha$-subunit from the $G \beta \gamma$ subunit of $G$ proteins. The guanine nucleotide-binding protein $\mathrm{G}(\mathrm{s})$ subunit alpha isoforms short (GNAS, Gos) class directly catalyzes the transformation of ATP to cAMP. cAMP is responsible for the melanogenic actions of ligands such as $\alpha-\mathrm{MSH}$, including the activation of tyrosinase in melanin biosynthesis (12). The present study first
Table II. Genotyping records of the F2 and F3 generation mice.

\begin{tabular}{llll}
\hline Mouse ID no. & Genotyping & Mouse ID no. & Genotyping \\
\hline F2-2 & Wild-type & F3-4 & Wild-type \\
F2-7 & Wild-type & F3-5 & Wild-type \\
F2-17 & Wild-type & F3-7 & Wild-type \\
F2-3 & Homozygous & F3-10 & Wild-type \\
F2-13 & Homozygous & F3-14 & Homozygous \\
F2-1 & Heterozygous & F3-1 & Heterozygous \\
F2-4 & Heterozygous & F3-2 & Heterozygous \\
F2-5 & Heterozygous & F3-3 & Heterozygous \\
F2-6 & Heterozygous & F3-6 & Heterozygous \\
F2-8 & Heterozygous & F3-8 & Heterozygous \\
F2-9 & Heterozygous & F3-9 & Heterozygous \\
F2-11 & Heterozygous & F3-11 & Heterozygous \\
F2-12 & Heterozygous & F3-12 & Heterozygous \\
F2-16 & Heterozygous & F3-13 & Heterozygous \\
F2-18 & Heterozygous & F3-15 & Heterozygous \\
F2-19 & Heterozygous & F3-16 & Heterozygous \\
\hline
\end{tabular}

assessed the expression of the human SASH1 protein or mouse Sash1 protein in wild-type, homozygous and heterozygous mice using the Novus SASH1 antibody (cat. no. NBP-26650, Novus Biologicals, LLC), which can recognize both the human SASH1 and mouse Sash1 proteins. The results of western blot analysis indicated that in the F2 and F3 generations, unlike the SASH1 or Sashl gene expressed at the transcriptional level, SASH1 or Sash1 protein was expressed in the majority of the heterozygous mice and wild-type mice. However, in a few heterozygous mice and wild-type mice, SASH1 or Sash1 protein were not detected (Figs. 3C and F, and 4A and D). The expression of SASH1 protein in the $\mathrm{F} 2$ and $\mathrm{F} 3$ generations was compared, and statistical analyses suggested that there were no significant differences between the F2 and F3 generations (Fig. 4G).

In the present study, in $27.3 \%(3 / 11)$ of the heterozygous mice with the Y551D-SASH1 mutation in the F2 generation, GNAS expression in the examined mice was increased compared with that in all 3 wild-type mice (Fig. 3C and D). The total expression of GNAS in the 11 heterozygous mice of the F2 generation was also increased compared with that in the 3 wild-type mice of the F2 generation (Fig. 3C and E). However, in 10 heterozygous mice with the Y551D-SASH1 mutation in the F3 generation, GNAS expression was not induced compared with that in 4 wild-type mice (Fig. 3F and G). The total expression of GNAS in 10 heterozygous mice of the F3 generation was also not induced compared with that in 4 wild-type mice (Fig. 3F and $\mathrm{H}$ ).

In the F2 generation, the expression of GNAS was significantly decreased in most heterozygous mice compared with the homozygous mice (Fig. 4A and B), and the total expression of GNAS in the 11 heterozygous mice was significantly decreased compared with the homozygous mice (Fig. 4A and C). In $81.8 \%$ (9/11) of the heterozygous mice in the F3 generation, GNAS expression in the examined mice was also upregulated compared with that in the F3-14 homozygous 

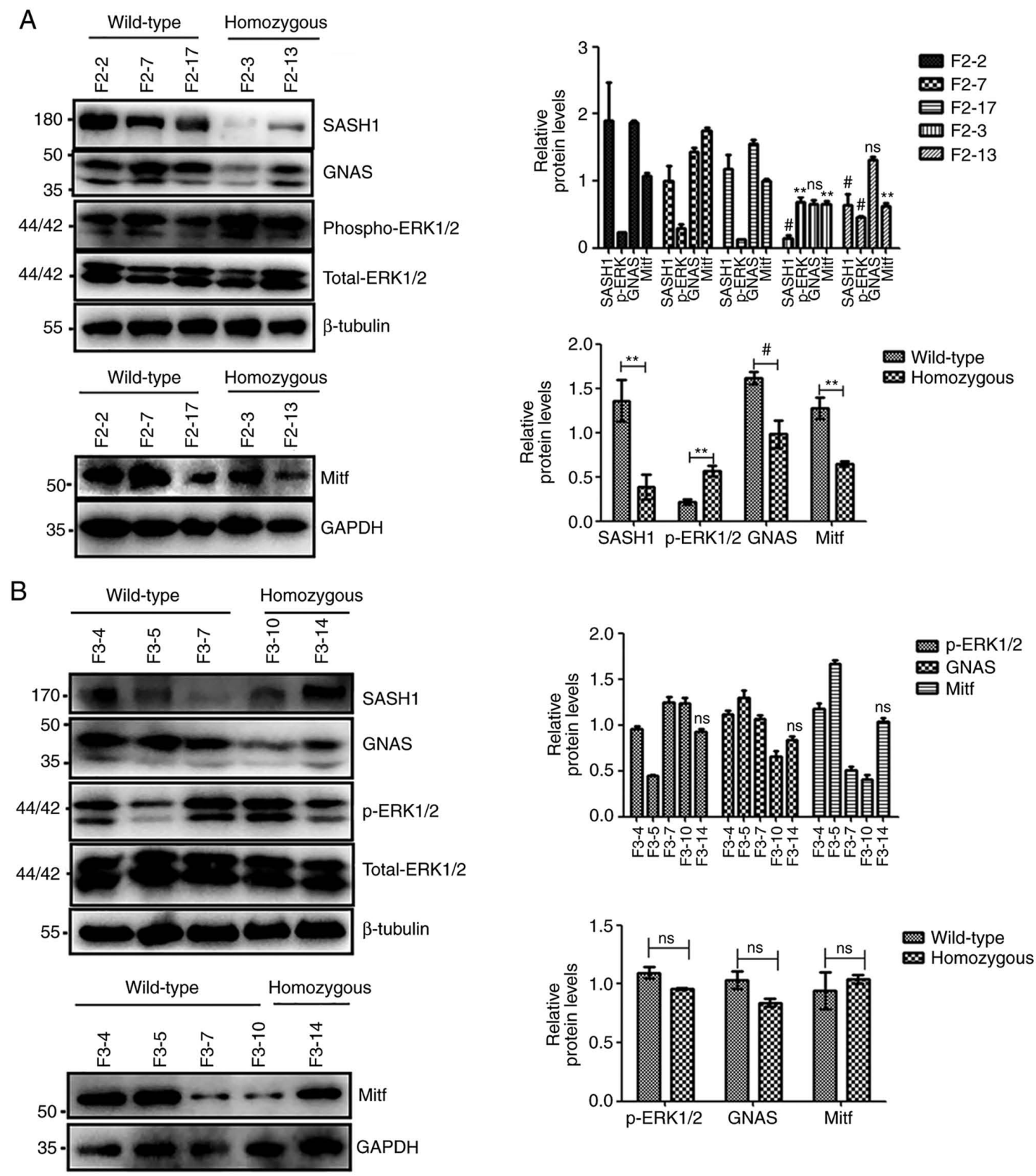

Figure 3. Mitf, but not GNAS, and phospho-ERK1/2 were uniformly increased in heterozygous hSASH1(Y551D) gene knock-in BABL/cJ mice compared to wild-type mice. (A) Downregulation of SASH1 was induced by the Y551D SASH1 mutation in homozygous mice of the F2 generation compared to that of wild-type mice. GNAS and Mitf expression was attenuated by the downregulation of SASH1; however, phospho-ERK1/2 expression was increased (left panel). The total densitometry values of these proteins were also compared collectively (lower right panel). Upper right panel: ${ }^{*} \mathrm{P}<0.01 \mathrm{vs}$. all 3 wild-type mice, ${ }^{* *} \mathrm{P}<0.001$ vs. all 3 wild-type mice; ns, not significant vs. all 3 wild-type mice. Lower right panel: ${ }^{\#} \mathrm{P}<0.01$, homozygous mice vs. wild-type mice, ${ }^{* *} \mathrm{P}<0.001$ vs. all 3 wild-type mice. (B) SASH1, GNAS and Mitf expression was not attenuated by the Y551D-SASH1 mutation in the F3 generation, as indicated by western blot and statistical analyses. ns: No significance vs. all four wild-type mice.

mouse (Fig. 4D and E). The total expression of GNAS in the 11 heterozygous mice of the F3 generation was also upregulated compared to that in the 1 homozygous mouse (Fig. 4D and F). In addition, obvious differences were observed in GNAS expression between the wild-type mice and homozygous mice of the F2 generation (Fig. 3A). However, no marked differences were observed in GNAS expression between the $\mathrm{F} 3$ generation mice (Fig. 3B).

Phospho-ERK1/2 is not uniformly induced in heterozygous hSASH1(Y551D) gene knock-in BABL/cJ mice. Mitf is phosphorylated by upstream ERK and induces increased transcriptional activity of the tyrosinase promoter (13-15). A previous study by the authors demonsrtated that SASH1 was induced by the p53-POMC-MC1R signal cascade to enhance the phosphorylation of ERK1/2 and CREB and that mutated SASH1 alleles promoted increased protein levels of phosphorylated ERK1/2 and CREB (4). The ratio of phospho-ERK1/2:ERK1/2 (p-ERK/ERK) was calculated when assessing the effects of expression of human Y551D SASH1 or mouse Sash1 on that of phospho-ERK1/2 in F2 and F3 generation. The present study found that the ratio of p-ERK/ERK was significantly increased in $90.9 \%$ (10/11) of the heterozygous mice compared to the wild-type mice in the 

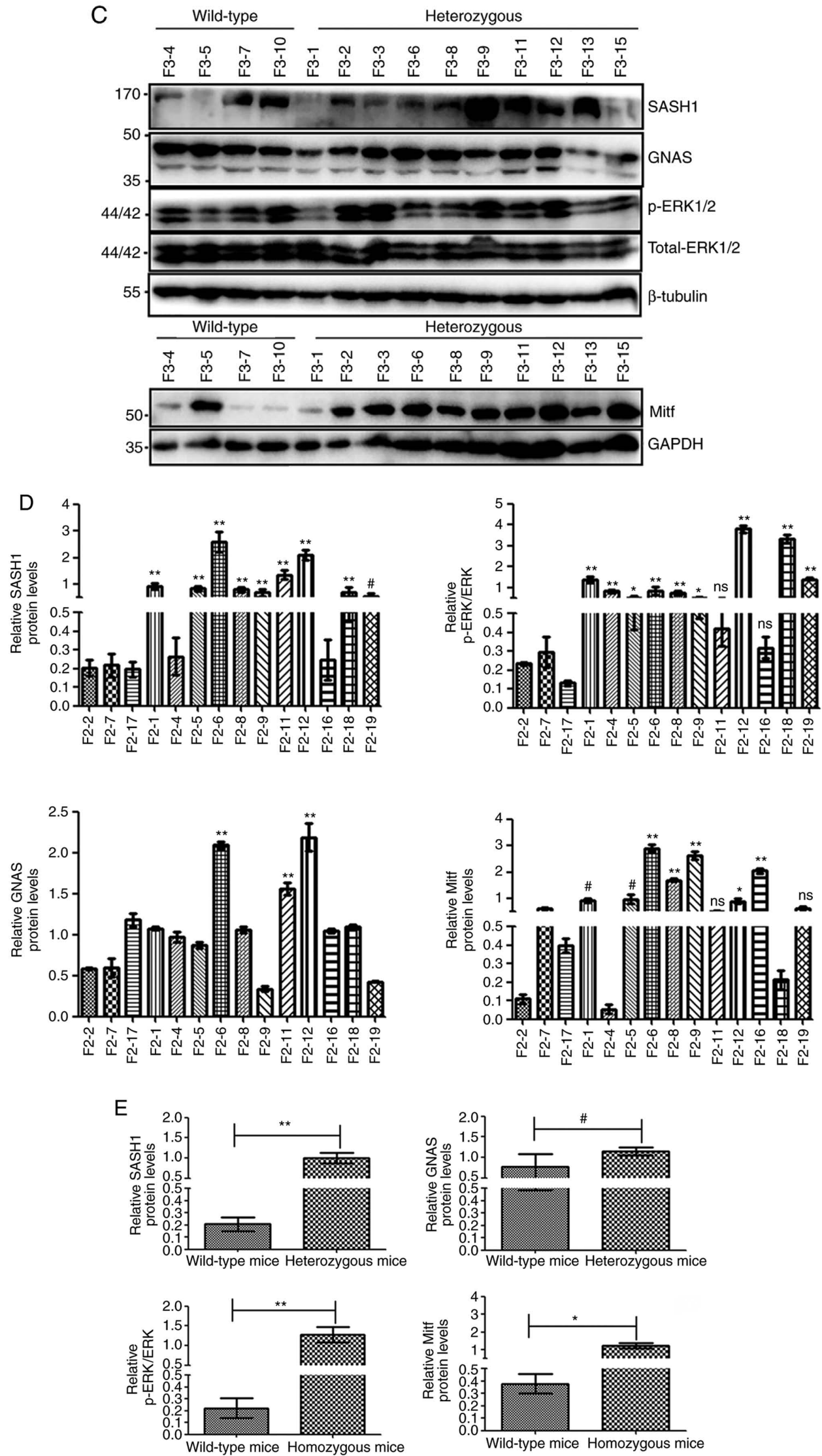

Figure 3. Continued. Mitf, but not GNAS, and phospho-ERK1/2 were uniformly increased in heterozygous hSASH1(Y551D) gene knock-in BABL/cJ mice compared to wild-type mice. (C-E) Tail biopsies of wild-type mice and heterozygous mice of the F2 generation were lysed, ultrasonicated and subjected to western blot analysis. The results of western blot analysis indicated that the expression of SASH1, GNAS, phospho-ERK1/2 and Mitf was enhanced in the heterozygous hSASH1(Y551D) gene knock-in BABL/cJ mice of the F2 generation compared to wild-type mice. (D) The densitometry values of SASH1, GNAS, phospho-ERK1/2 and Mitf of 11 heterozygous and 3 wild-type mice were compared, and (E) the total densitometry values of these proteins were also compared collectively. (D) "P $<0.05$ vs. all 3 wild-type mice, ${ }^{\prime \prime} \mathrm{P}<0.01$ vs. all 3 wild-type mice, ${ }^{* *} \mathrm{P}<0.001$ vs. all 3 wild-type mice. (E) ${ }^{*} \mathrm{P}<0.05$ vs. wild-type mice; ${ }^{\#} \mathrm{P}<0.01$ vs. wild-type mice, ${ }^{* *} \mathrm{P}<0.001$ vs. wild-type mice. 

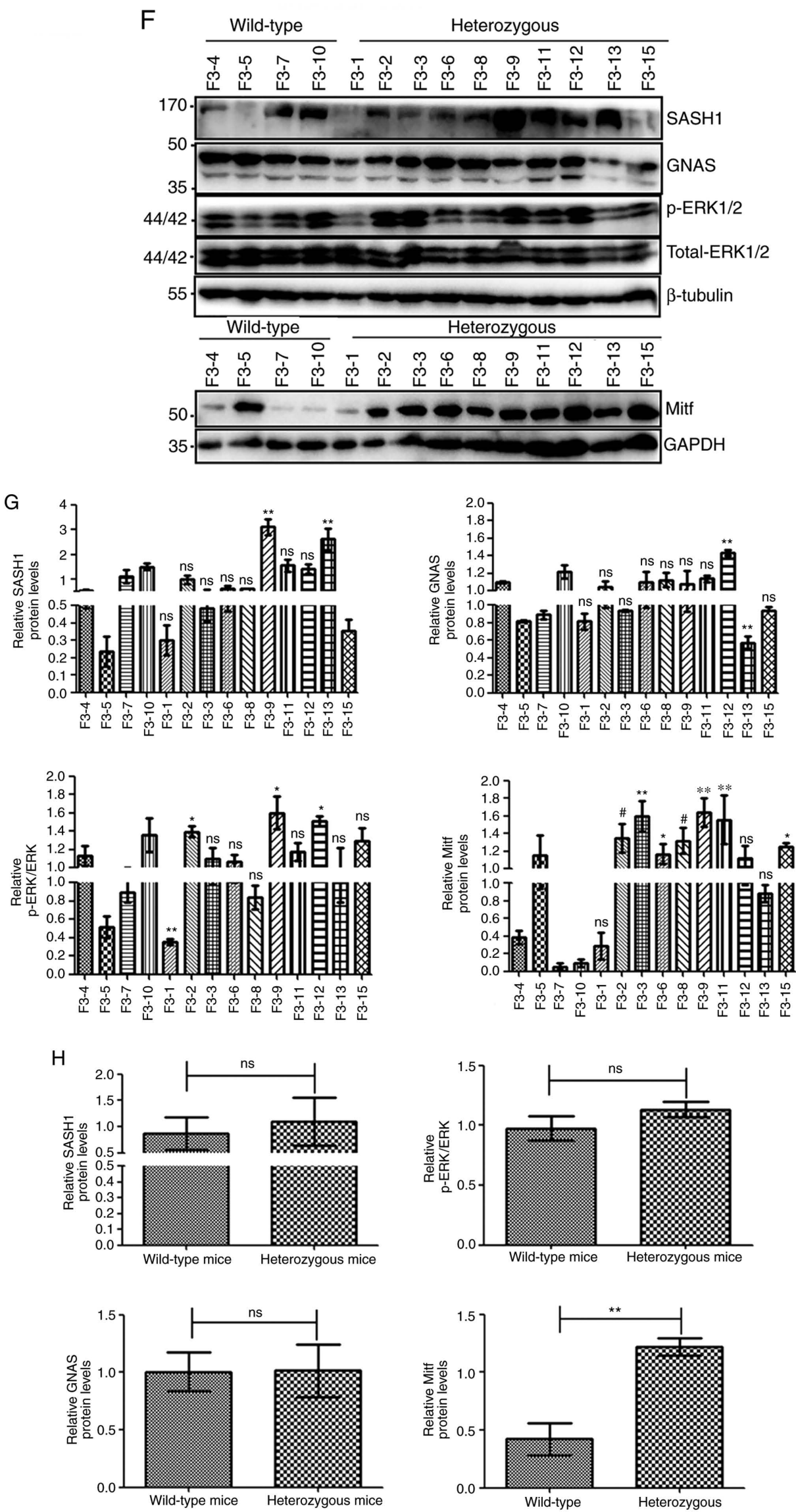

Figure 3. Continued. Mitf, but not GNAS, and phospho-ERK1/2 were uniformly increased in heterozygous hSASH1(Y551D) gene knock-in BABL/cJ mice compared to wild-type mice. (F-H) Western blot analysis revealed that the expression of Mitf was enhanced in heterozygous human Y551D SASH1 gene knock-in mice of the F3 generation compared with wild-type mice. (G) The densitometry values of SASH1, GNAS, phospho-ERK1/2 and Mitf of 10 heterozygous and 4 wild-type mice were compared, and $(\mathrm{H})$ the total densitometry values of these proteins were also compared collectively. (G) ${ }^{*} \mathrm{P}<0.05$ vs. all 3 wild-type mice, ${ }^{\#} \mathrm{P}<0.01$ vs. all 3 wild-type mice; ${ }^{* *} \mathrm{P}<0.001$ vs. all 3 wild-type mice. $(\mathrm{H}){ }^{* * *} \mathrm{P}<0.001$ vs. wild-type mice. For all panels, ns, not significant. 


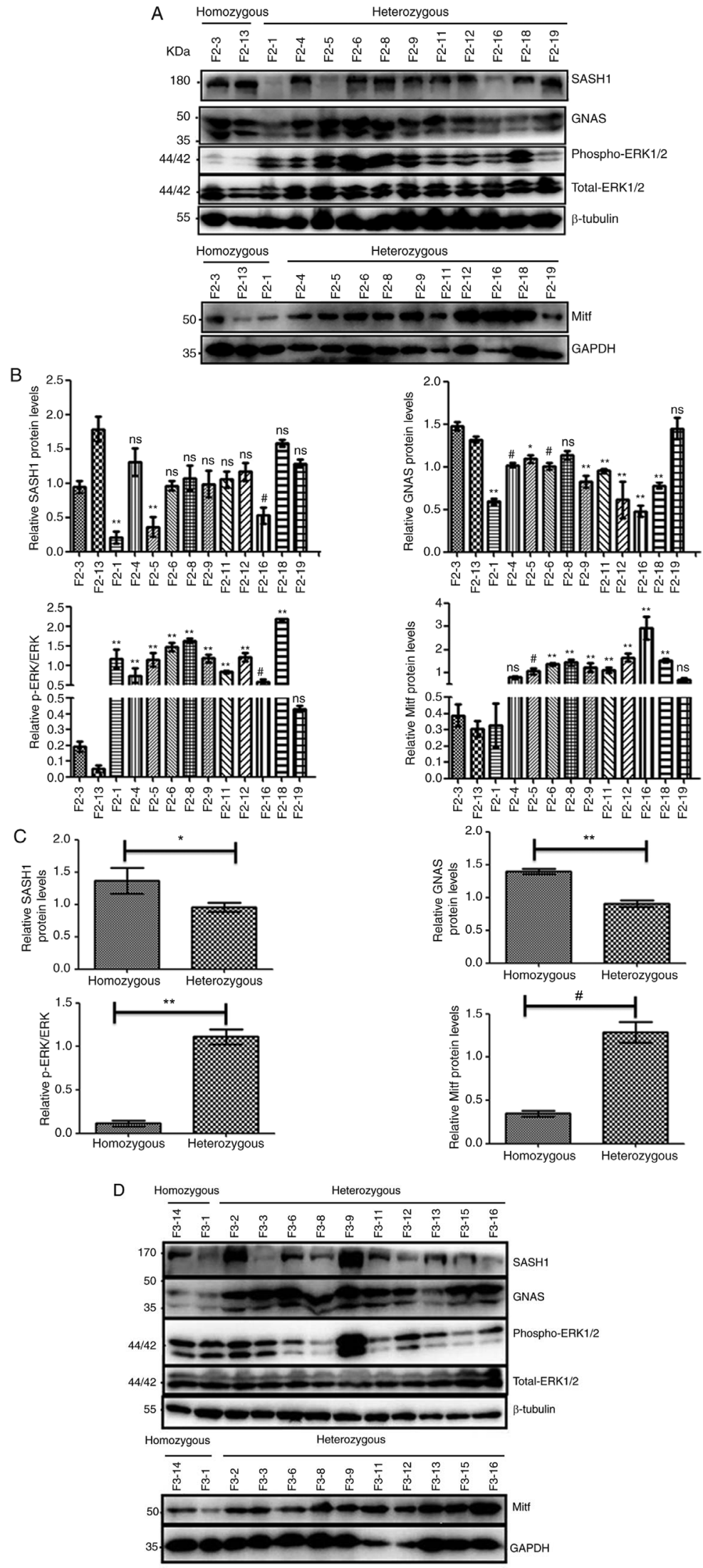

Figure 4. Mitf, but not GNAS, and phospho-ERK1/2 expression is increased in heterozygous human Y551D SASH1 knock-in mice compared to homozygous mice. (A) Western blot analysis indicated that SASH1 was downregulated in heterozygous human Y551D-SASH1- knock-in mice of the F2 generation compared to homozygous mice. However, the protein levels of phospho-ERK1/2 and Mitf were upregulated in heterozygous mice compared to those of homozygous mice. (B and C) The densitometry values of SASH1, GNAS, phospho-ERK1/2 and Mitf of 11 heterozygous and 2 homozygous mice of the F2 generation were compared, and the total densitometry values of these proteins were also compared collectively, respectively. (B) "P<0.05 vs. both homozygous mice, ${ }^{"} \mathrm{P}<0.01$ vs. both homozygous mice; ${ }^{* *} \mathrm{P}<0.001$ vs. both homozygous mice. (C) ${ }^{\#} \mathrm{P}<0.01$ vs. homozygous mice, ${ }^{* *} \mathrm{P}<0.001$ vs. homozygous mice, ${ }^{*} \mathrm{P}<0.05$ vs. homozygous mice. (D) Western blot analysis demonstrated that the expression of Mitf and GNAS was enhanced in heterozygous human Y551D SASH1 knock-in mice of the F3 generation compared to homozygous mice. 

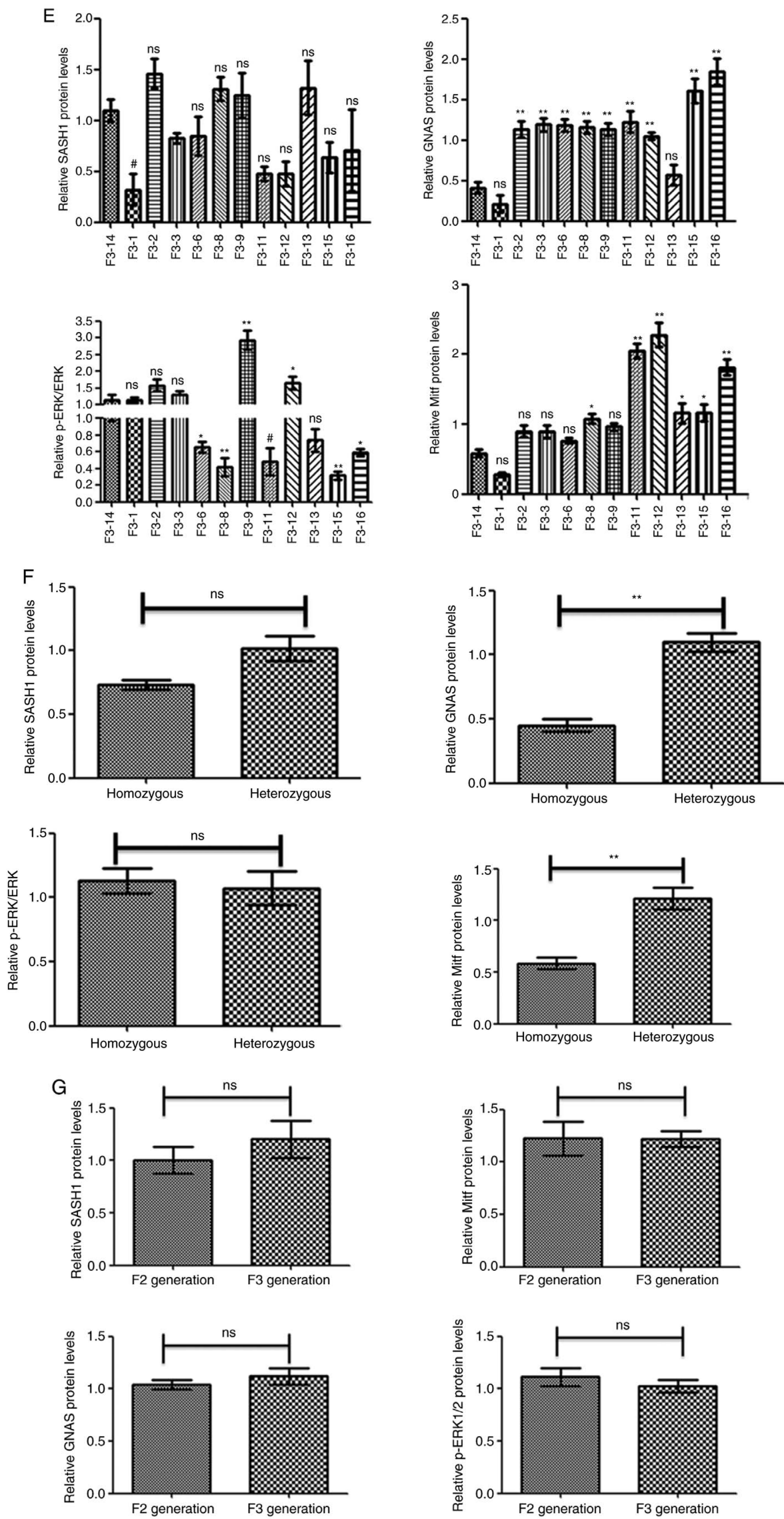

Figure 4. Continued. Mitf, but not GNAS, and phospho-ERK1/2 expression is increased in heterozygous human Y551D SASH1 knock-in mice compared to homozygous mice. (E and F) The densitometry values of SASH1, GNAS, phospho-ERK1/2 and Mitf of 11 heterozygous and 1 homozygous F3 generation mice were compared. (F) The total gray values of these proteins were also compared collectively. (E) "P<0.05 vs. one homozygous mouse, respectively, ${ }^{*} \mathrm{P}<0.01$ vs. one homozygous mouse, respectively, ${ }^{* *} \mathrm{P}<0.001$ vs. one homozygous mouse, respectively. $(\mathrm{F}){ }^{* * *} \mathrm{P}<0.001$ vs. homozygous mice. (G) The densitometry values of SASH1, Mitf, GNAS and the ratio of p-ERK/ERK in 11 heterozygous mice of the F2 generation and 10 heterozygous mice of the F3 generation were compared. For all panels, ns, not significant. 


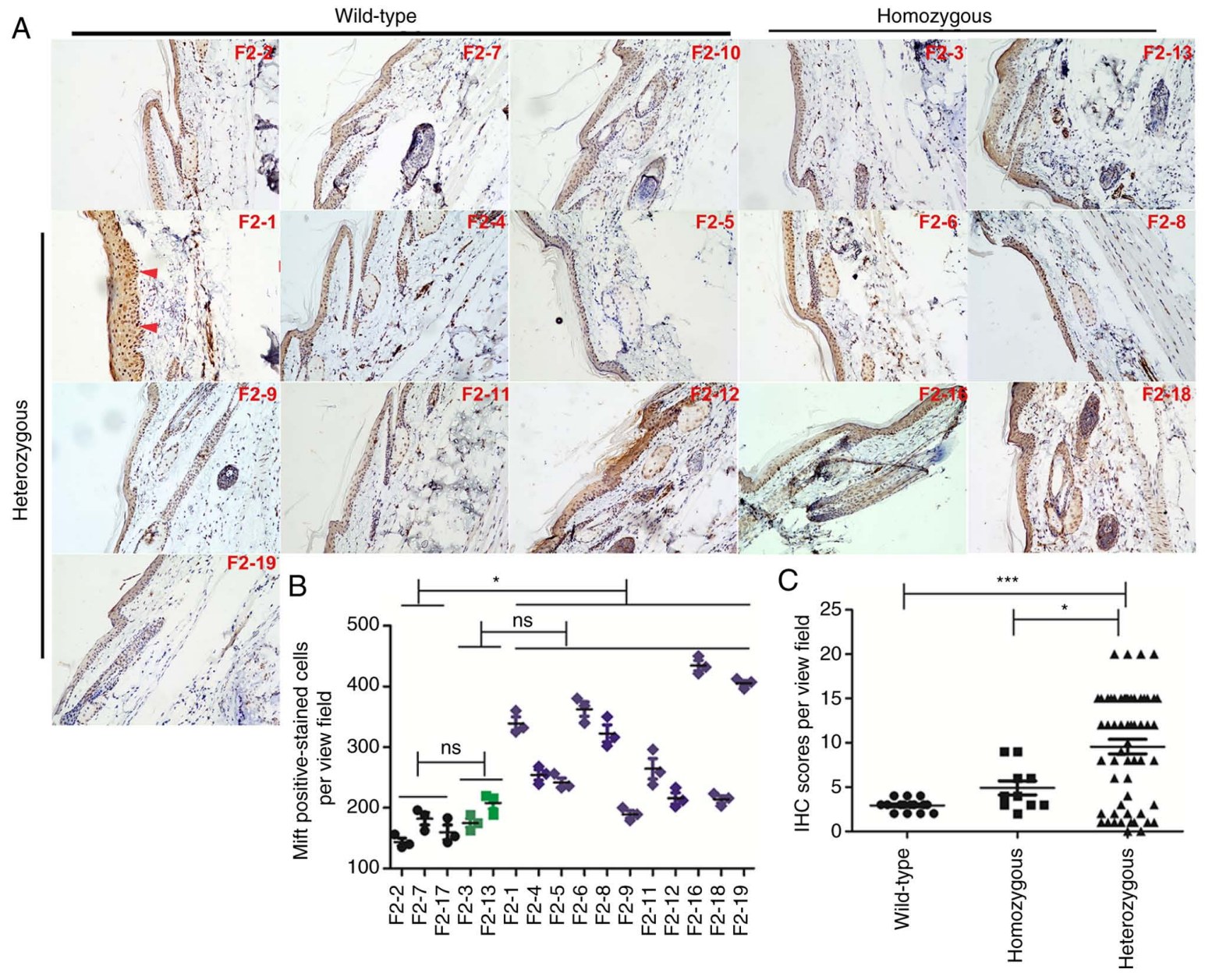

Figure 5. In the F2 generation, the number of Mitf- and Mitf-positive epithelial cells was enhanced in heterozygous human Y551D SASH1 knock-in mice. (A) Representative images (magnification, $\mathrm{x} 10$ ) of Mitf in 3 wild-type mice, 2 homozygous mice and 11 heterozygous mice. (B) A total of 5 random visual fields in each section of 16 mice, including wild-type, homozygous and heterozygous mice, were photographed. The Mitf-positive epithelial cells in the tail tissues of wild-type, homozygous and heterozygous mice were counted and analyzed statistically. Representative Mitf-positive cells, which were stained dark brown in the nucleus, are indicated by red arrows. ${ }^{*} \mathrm{P}<0.01$. (C) The staining intensity and percentage of Mitf-positive cells per mouse were calculated, scored and analyzed statistically. $\mathrm{P}<0.05,{ }^{* * *} \mathrm{P}<0.001 ;$ ns, not significant.

F2 generation (Fig. 3C and D). The total ratio of p-ERK/ERK in the 11 heterozygous mice was also significantly increased compared with that of the wild-type mice (Fig. 3C and E). However, only 3 marked increases in the ratio of p-ERK/ERK among 11 heterozygous mice were observed compared with the wild-type mice in the F3 generation, when the phospho-ERK1/2 levels were compared separately (Fig. 3F and G).

The ratio of p-ERK/ERK was markedly enhanced in $100 \%$ (11/11) of the heterozygous mice of the F2 generation compared with both homozygous mice (F2-3 and F2-13) (Fig. 4A and B). Overall, the ratio of p-ERK/ERK was significantly increased in the heterozygous mice compared with the homozygous mice (Fig. 4A and C) of the F2 generation. In the heterozygous mice of the F3 generation, the ratio of p-ERK/ERK was increased only in $18.2 \%(2 / 11)$ of the heterozygous mice of the F3 generation compared with the homozygous mice (Fig. 4D and E). The total ratio of p-ERK/ERK was not markedly increased in the heterozygous mice compared to the homozygous mice (Fig. 4D and F) of the F3 generation.

Mitf expression and Mitf-positive-stained epithelial cells were uniformly enhanced in tail tissues of heterozygous human
SASH1(Y551D) gene-knock in BABL/cJ mice. Mitf contains both basic helix-loop-helix and leucine zipper structural features and is a melanocyte master transcription factor and a specific marker of melanocytes. Mitf regulates melanocyte development and is responsible for pigment cell-specific transcription of melanogenic enzyme genes (16). In a previous study, it was demonstrated that the downstream melanogenic enzymes of Mitf were induced by mutated SASH1 in the affected epithelial tissues and normal human epithelial melanocytes $(3,4)$. SASH1 not only localizes to the cytoplasm as previously indicated (2-4), but also localizes to the nucleus in epithelial cells (10). Mitf functions as the master regulator of the melanocytic lineage and is constitutively nuclear or translated to the nucleus $(17,18)$. Therefore, the present study evaluated whether SASH1 can assemble Mitf and whether mutated SASH1 can mediate Mitf expression.

The effects of SASH1 mutations on Mitf in hSASH1(Y551D) gene knock-in BABL/cJ mice were further identified. In the homozygous mice of the F2 generation, a decreased Mitf expression was induced in the homozygous human Y551D-SASH1 gene knock-in mice compared with the wild-type mice (Fig. 3A). However, Mitf expression was not 


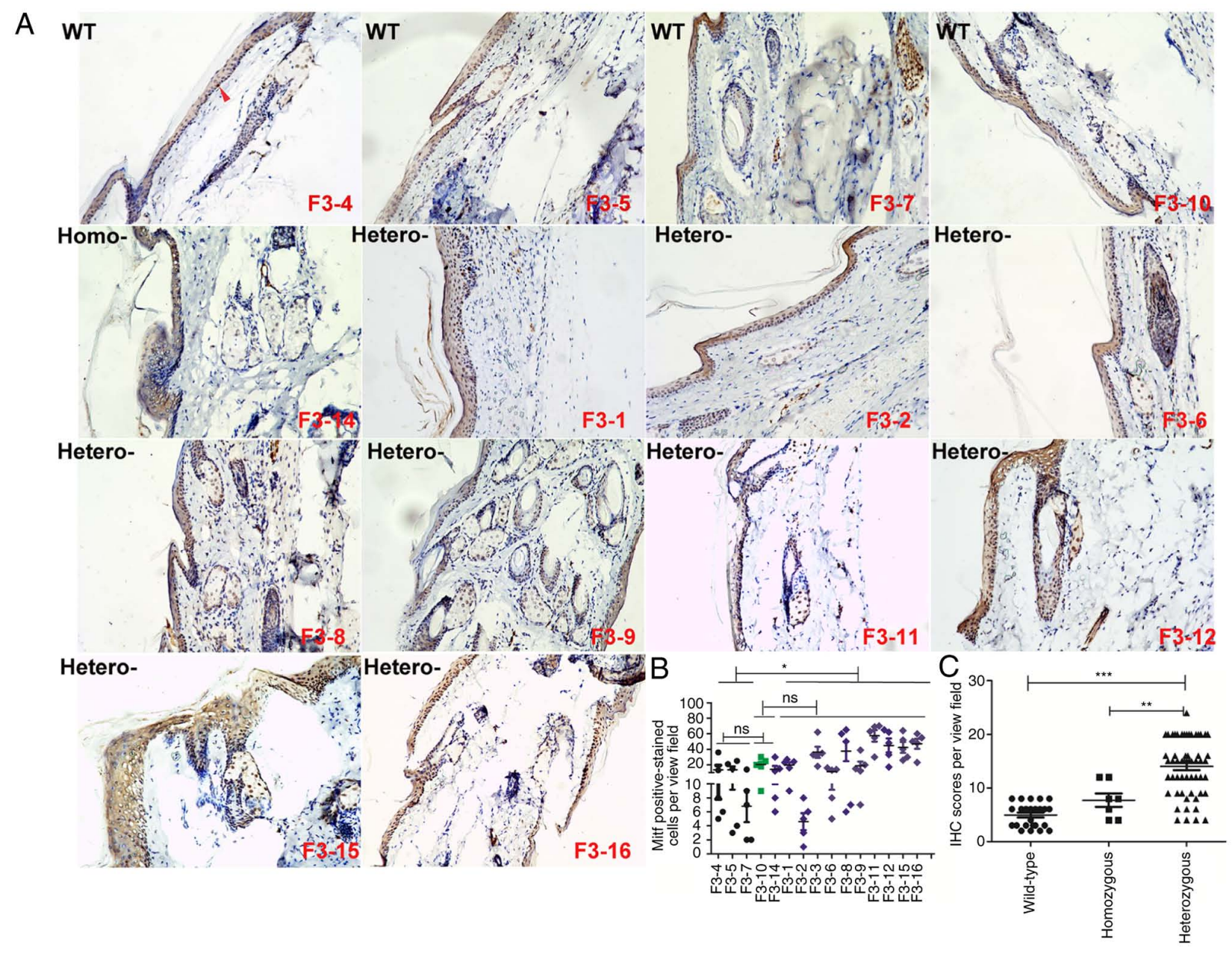

Figure 6. In the F3 generation, the number of Mitf- and Mitf-positive epithelial cells was augmented in heterozygous human Y551D SASH1 knock-in mice. (A) Representative images (magnification, x10) of Mitf in 4 wild-type mice, 1 homozygous mouse and 11 heterozygous mice. (B) A total of 51 visual fields in each section of 15 mice, including wild-type, homozygous and heterozygous mice, were photographed. The Mitf-positively stained epithelial cells in the tail tissues of wild-type, homozygous and heterozygous mice were calculated in 3 visual fields and analyzed statistically. Representative Mitf-positive cells, which were stained yellowish-brown in the nucleus, are indicated by red arrows. "P<0.01. (C) Staining intensity and percentage of Mitf-positive cells per mouse were calculated, scored and analyzed statistically. ${ }^{* *} \mathrm{P}<0.01^{* * * *} \mathrm{P}<0.001 ;$ ns, not significant.

downregulated in the homozygous mice of the F3 generation compared with the wild-type mice (Fig. 3B). In 63.6\% (7/11) of the heterozygous mice with the Y551D-SASH1 mutation of the F2 generation, an increased Mitf expression in the examined mice was induced compared with that in all 3 wild-type mice (Fig. 3C and D). The total expression of Mitf in the 11 heterozygous mice of the F2 generation was also enhanced compared to that in the 3 wild-type mice of the F2 generation (Fig. 3C and E). In 60\% (6/10) of the heterozygous mice with the Y551D-SASH1 mutation of the F3 generation, Mitf expression in the examined mice was enhanced compared with that in the 4 wild-type mice (Fig. 3F and G). The overall expression of Mitf in the 10 heterozygous mice of the F3 generation was also enhanced compared to that in the 4 wild-type mice (Fig. 3F and H).

The expression of Mitf was upregulated in the examined mice in $72.7 \%(8 / 11)$ of the heterozygous mice of the F2 generation compared with F2-3 and F2-13 homozygous mice (Fig. 4A and B). The expression of Mitf in the 11 heterozygous mice of the F2 generation was also increased compared with that in the 2 homozygous mice of the $\mathrm{F} 2$ generation (Fig. 4A and C). In 60\% (6/10) of the heterozygous mice of the F3 generation, Mitf expression in the examined mice was also upregulated compared with that in the F3-14 homozygous mouse (Fig. 4D and E). The overall expression of Mitf in the 11 heterozygous mice of the F3 generation was also upregulated compared with that in the 1 homozygous mouse (Fig. 4D and F). Taken together, these data indicated that there was a fluctuating Mitf expression between the wild-type mice and homozygous mice; however, a significant difference in Mitf expression was observed between the heterozygous mice and homozygous mice or the wild-type mice. No differences in the expression of GNAS and Mitf protein, as well as in the ratio of p-ERK/ERK were observed between the F2 and F3 generation mice (Fig. 4G).

Immunohistochemical analyses further demonstrated that the heterozygous mice of the F2 generation exhibited an increased expression of Mitf and increasing numbers of Mitf positively stained epithelial cells compared with those of the homozygous mice (Fig. 5A and B). The heterozygous mice also exhibited an increased expression of Mitf and increasing numbers of Mitf positively stained epithelial cells compared with those of wild-type mice (Fig. 5A and B). The overall comparisons of Mitf and the numbers of Mitf-positive 
A

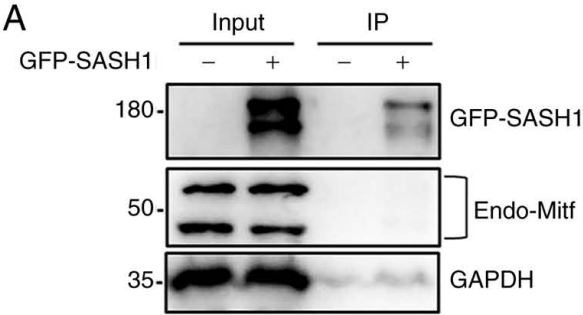

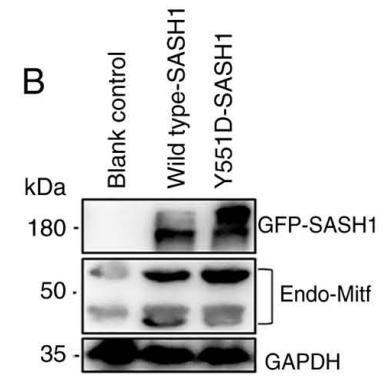

35
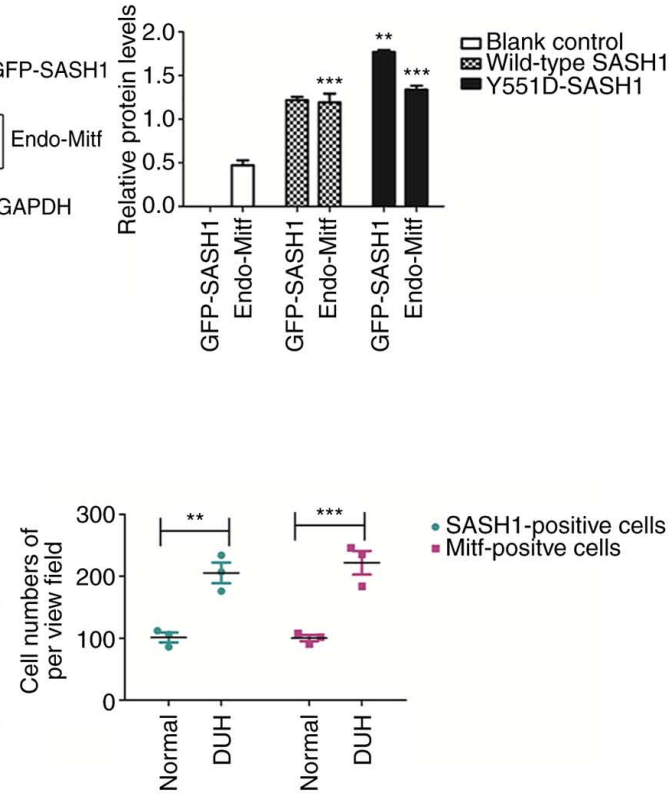

C

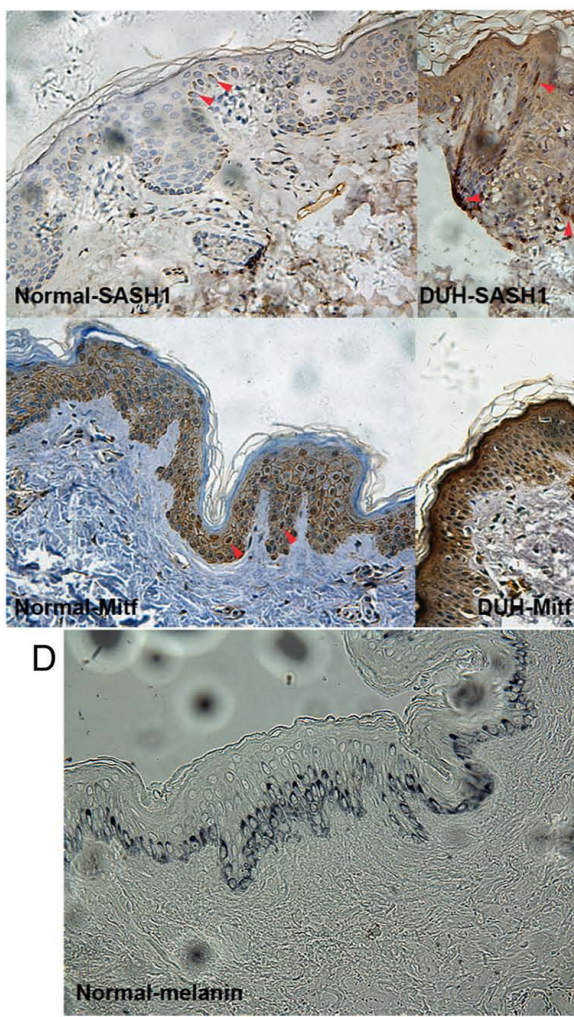

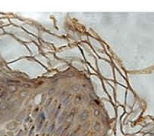
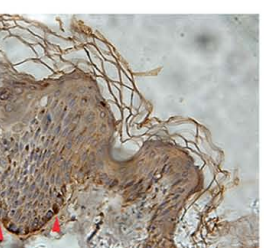
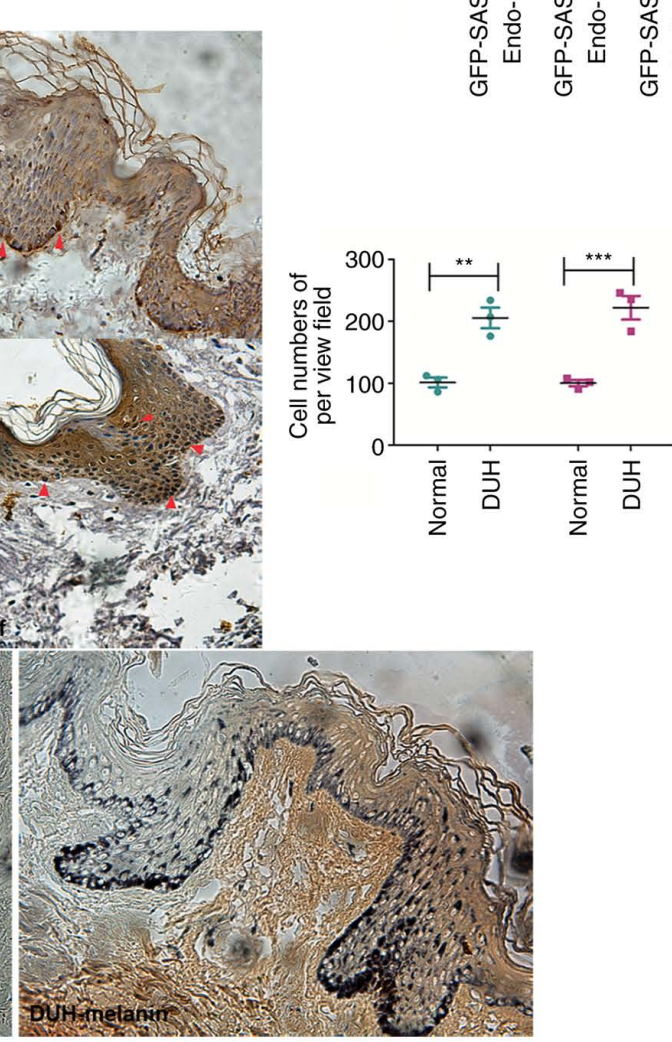

Figure 7. Mitf expression is promoted by mutated SASH1 in vitro and in epithelial tissues affected by the Y551D-SASH1 mutation. (A) Exogenous SASH1 is not associated with endogenous Mitf in 293T cells. GFP-SASH1 was transfected into 293T cells. At 48 h following transfection, transfected cells were lysed, GFP-SASH1 was immunoprecipitated, and the associated endogenous Mitf was analyzed by IP-WB analyses. (B) Expression of endogenous Mitf was induced by Y551D SASH1. Exogenous Y551D SASH1 and a wild-type SASH1 were introduced into 293T cells. Following transfection, transfected cells were lysed and subjected to western blot analyses. ${ }^{* *} \mathrm{P}<0.001$ vs. wild-type SASH1 and ${ }^{* * *} \mathrm{P}<0.001$ vs. blank control. (C) In the lesional epithelial tissues of Y551D SASH1-affected individuals, SASH1- and Mitf-positive cells were demonstrated in different epithelial layers of the affected epithelial tissues and calculated and analyzed statistically. ${ }^{* *} \mathrm{P}<0.01,{ }^{* * *} \mathrm{P}<0.001$. Upregulation of SASH1 and enhanced Mitf were also induced in the affected tissues. Magnification, $\mathrm{x} 40$. Mitf-positive cells, which were stained dark brown in the nucleus, are indicated by red arrows. (D) More melanin was synthesized and present in different epithelial layers of the affected skin epithelial tissues. Magnification, $\mathrm{x} 40$.

epithelial cells between the wild-type and heterozygous mice, or between the homozygous and heterozygous mice were also performed. Statistical analyses indicated that the overall expression of Mitf and the numbers of Mitf-positive epithelial cells of the heterozygous mice of the F2 generation were increased compared with those of the wild-type and homozygous mice (Fig. 5B and C).

The enhanced expression of Mitf and increasing numbers of Mitf-positive epithelial cells were observed in the examined heterozygous mice of the F3 generation compared with those of the homozygous mice or wild-type mice (Fig. 6A and B). Statistical analyses indicated that the overall expression of Mitf in the heterozygous mice of the F3 generation was increased compared with that in the wild-type and homozygous mice (Fig. 6B and C).
Mitf expression and Mitf-positive epithelial tissues are increased in the affected tissues from Y551D-SASH1-affected patients. The above-mentioned results indicated that Mitf expression was increased in the heterozygous human SASH1 (Y551D) gene knock-in BABL/cJ mice. Therefore, it was hypothesized that SASH1 may be associated with Mitf. Therefore, IP-WB analyses were performed to identify the associations between exogenous SASH1 and endogenous Mitf. However, IP-WB analyses indicated that GFP-SASH1 did not bind to endogenous Mitf in 293T cells (Fig. 7A). Subsequently, whether endogenous Mitf is mediated by exogenous SASH1 was further determined. The results of western blot analysis indicated that endogenous Mitf expression was increased by mutated SASH1 (Fig. 7B). The induction of Mitf by mutated SASH1 was assessed in the epithelial tissues of affected 
individuals. Immunohistochemical analyses indicated that more SASH1- and Mitf-positive epithelial cells were present in the affected tissues than in the normal controls. A higher expression of SASH1 and Mitf was also induced in the affected tissues (Fig. 7C). A increased amount of melanin was synthesized in different epithelial layers of the affected tissues (Fig. 7D).

\section{Discussion}

DUH was initially described in 2 generations of 2 families in 1933 (19) and is a group of congenital pigmentary disorders characterized by generalized mottled hypopigmented and hyperpigmented macules $(5,20)$. DUH clinically overlaps with DSH and Dowling-Degos disease and genetically exhibits heterogeneity with at least 2 causative genes, ABCB6 and SASH1 $(5,8,20)$. In the present study, a heterozygous mSash- $h S A S H 1$ (Y551D) gene knock-in BALB/cJ mouse model was established to recapitulate some molecular pathological phenotypes of DUH. SASH1 may function as a scaffold protein for the assembly Mitf in the nucleus, generating a novel SASH1-Mitf signaling pathway to regulate melanogenesis in vitro and in vivo. Y551D SASH1 promotes Mitf expression and increases the numbers of Mitf-positive epithelial cells in the affected human epithelial tissues and in the tail tissues of heterozygous mice; this phenomenon contributes to the formation of the hyperpigmentation phenotype of DUH.

SASH1, a signaling adaptor protein of 1,247 amino acids, is located on chromosome 6q24.3 and contains an evolutionarily conserved SLY domain (401-555), an SH3 domain (557-614) and two SAM domains (633-697; 1177-1241, annotation from the UniProt database) (5). The SASH1 gene, which functions as a potential tumor suppressor, has been reported to be associated with the tumorigenesis of lung cancer, breast cancer, colon cancer, cervical cancer, melanoma and hepatocellular carcinoma cells and is inversely associated with certain critical ultrasonographic features of breast cancer $(5,9,21-23)$. In the present study, the human SASHl gene or mouse Sashl gene was expressed at the transcriptional level (Fig. 2C). However, in a few heterozygous and wild-type mice, the human SASH1 protein or mouse Sash1 protein was not detected. The underlying reason for this may be due to the post-translational modification of SASH1 as an RXRXXpS phosphorylated motif was identified in SASH1; SASH1 is suggested to be phosphorylated at Ser90 (24), and multiple serine sites are demonstrated in the PhosphoSitePlus website (https://www.genecards.org/Product Redirect?key=fAAAAAJnAAYAAABTQVNIMQAQdAATAAAAAnYABAAAAENTVAAKcwAIYgAAAnUARAA AAGh0dHBzOi8vd3d3LnBob3NwaG9zaXRlLm9yZy91bm lwcm90QWNjQWN0aW9uLmRvP2lkPU85NDg4NSNhcHBsZXRNc2cACm4ACmEAAA $\% 3 \mathrm{~d} \% 3 \mathrm{~d} \&$ section=proteins\&su bsection=). Therefore, it can be deduced that the undetectable SASH1 may be caused by the phosphorylational degradation of SASH1.

In a previous study by the authors, it was demonstrated that SASH1 mediated skin melanogenesis through a cascade of p53/ $\alpha-M S H / P O M C / G \alpha s / S A S H 1$ and novel SASH1/MAP2K2 crosstalk $(3,4)$. Increasing numbers of SASH1 variants have been found to be associated with human skin dyschromia, including DUH (2-5,9), multiple lentigines (6-8) and pigmentation defects with palmoplantar keratoderma and skin carcinoma (9). Apart from 3 additional variants in SASH1 that were reported, which are located in the SLY domain of SASH1 (E509K, L515P, and Y551D), 5 of the 7 different SASH1 variants identified that result in skin dyschromia are located in the highly conserved SLY domain $(5,7,8)$. All these findings suggest that the SLY domain is functionally critical for skin pigmentation regulation and may represent a potential mutational hotspot region (5). Recent research indicates that endothelial SASH1 regulates alveolar epithelial cell maturation and promotes pulmonary surfactant production through nitric oxide signaling (25). SASH1 is expressed in a number of human tissues and cells, including whole skin, keratinocytes, fibroblasts and melanocytes (NCBIGene Expression Omnibus; http://www.ncbi.nlm.nih.gov/geo/) (6). SASH1 expression has also been detected in cultured human epidermal melanocytes $(3,4)$, and the exogenous human Y551D SASH1 introduced into BALB/cJ mice did not affect the expression of endogenous $S a s h 1$ in mice as the endogenous Sash1 protein in mice can be recognized by the SASH1 antibody (NBP1-26650, Novus Biologicals, LLC). The S519N substitution in SASH1 was indicated to increase the number of melanocytes and epidermal cell proliferation in skin (6). In a previous study by the authors (4), and in the present study, it was suggested that the Y551D SASH1 substitution not only enhances Mitf expression, but also increases the numbers of Mitf-positive cells in the epithelial tissue of heterozygous human SASH1(Y551D) gene knock-in BABL/cJ mice and in Y551D affected individuals. Mitf mediates the proliferation of melanocytes and regulates pigment cell-specific transcription of melanogenic enzyme genes (16). Hence, the Y551D SASH1 substitution plays a critical role in the formation of the hyperpigmented phenotype of DUH.

The phosphorylated active form of CREB binds and activates Mitf (14,26-30). Mitf in turn stimulates the transcription of key melanogenic enzymes, such as tyrosinase, tyrosinase-related protein-1 and tyrosinase-related protein-2. Mitf plays a central role in $\alpha$-MSH-induced melanogenesis, and its expression and activation are regulated in a complex manner (31). In the present study, it was found that in heterozygous mice of 2 generations and in Y551D-affected skin epithelial tissues, Mitf was upregulated by Y551D-SASH1 in mice and in human tissues. Although exogenous SASH1 was not associated with endogenous Mitf, endogenous Mitf was regulated by Y551D SASH1. SASH1 serves as a novel scaffold protein to assemble a signaling complex downstream of TLR4 (32) and oncoprotein cortactin (10). Therefore, as shown in the present study, SASH1 may function as a scaffold molecule to assemble a SASH1-Mitf molecular complex to regulate MITF expression in the cell nucleus. All these findings indicate that SASH1 and Mitf may form a novel SASH1-Mitf cascade to regulate melanogenesis.

\section{Acknowledgements}

The authors would like to thank the Clinical Research Center at the Affiliated Hospital of Guizhou Medical University and the Experimental Animal Center of Guizhou Medical University for housing the animals for the experiments. The authors would also like to thank the Nanjing Biomedical 
Research Institute of Nanjing University which assisted in the creation of the models of mSash-hSASH1(Y551D) gene knock-in BABL/cJ mice.

\section{Funding}

The present study was supported partly by the following funds: The National Natural Science Foundation Project (31860319 to DAZ), the Guizhou Provincial Natural Science Foundation [grant no. Qian Ke He LH (2017) 7193 and Qian Ke He Zhicheng (2020) 4Y125 to DAZ], the Basic Conditions Platform of Guizhou Provincial Natural Science Foundation [grant no. Qianke Platform (2015) 40005 to for PH] and the Guizhou Provincial Natural Science Foundation [grant no. Qianke LH (2015) 7445 to PH].

\section{Availability of data and materials}

The datasets used and/or analyzed during the present study are available from the corresponding author on reasonable request.

\section{Authors' contributions}

DAZ and JZ designed the study, analyzed the data, and wrote and revised the manuscript. ZX, DWa, DWu, JW and LC performed the majority of the experiments. YD, JZ, YL, ZW, $\mathrm{XW}, \mathrm{QL}$ and JZ participated in some of the experiments. HH and $\mathrm{PH}$ provided some suggestions as regards the construction of the mouse models and some funds to support the projects. All authors have read and approved the final manuscript.

\section{Ethics approval and consent to participate}

For the use of human samples, the document of patient consent had been signed by the affected individual and 2 normal controls, and ethics approval was provided by the Ethics Committee of the Affiliated Hospital of Guizhou Medical University. All animal studies were conducted according to experimental practices and standards approved by the Ethics Committee of the Nanjing Biomedical Institute of Nanjing University and Guizhou Medical University (License no. 1800125).

\section{Patient consent for publication}

Not applicable.

\section{Competing interests}

The authors declare that they have no competing interests.

\section{References}

1. Xing QH, Wang MT, Chen XD, Feng GY, Ji HY, Yang JD, Gao JJ, Qin W, Qian XQ, Wu SN and He L: A gene locus responsible for dyschromatosis symmetrica hereditaria (DSH) maps to chromosome 6q24.2-q25.2. Am J Hum Genet 73: 377-382, 2003.

2. Zhou D, Wei Z, Deng S, Wang T, Zai M, Wang H, Guo L, Zhang J, Zhong $\mathrm{H}$, He L and Xing Q: SASH1 regulates melanocyte transepithelial migration through a nove Gas-SASH1-IQGAP1-E-cadherin dependent pathway. Cell Signal 25: 1526-1538, 2013.
3. Zhou D, Wei Z, Kuang Z, Luo H, Ma J, Zeng X, Wang K, Liu B, Gong F, Wang J, et al: A novel P53/POMC/Gas/SASH1 autoregulatory feedback loop activates mutated SASH1 to cause pathologic hyperpigmentation. J Cell Mol Med 21: 802-815, 2017.

4. Zhou D, Kuang Z, Zeng X, Wang K, Ma J, Luo H, Chen M, Li Y, Zeng J, Li S, et al: p53 regulates ERK1/2/CREB cascade via a novel SASH1/MAP2K2 crosstalk to induce hyperpigmentation. J Cell Mol Med 21: 2465-2480, 2017.

5. Zhong WL, Wang HJ, Lin ZM and Yang Y: Novel mutations in SASH1 associated with dyschromatosis universalis hereditaria. Indian J Dermatol Venereol Leprol 85: 440, 2019.

6. Shellman YG, Lambert KA, Brauweiler A, Fain P, Spritz RA, Martini M, Janssen KP, Box NF, Terzian T, Rewers M, et al: SASH1 is involved in an autosomal dominant lentiginous phenotype. J Invest Dermatol 135: 3192-3194, 2015.

7. Wang J, Zhang J, Li X, Wang Z, Lei D, Wang G, Li J, Zhang S, $\mathrm{Li} \mathrm{Z}$ and $\mathrm{Li} \mathrm{M}$ : A novel de novo mutation of the SASH1 gene in a chinese family with multiple lentigines. Acta Derm Venereol 97: 530-531, 2017.

8. Zhang J, Cheng R, Liang J, Ni C, Li M and Yao Z: Lentiginous phenotypes caused by diverse pathogenic genes (SASH1 and PTPN11): Clinical and molecular discrimination. Clin Genet 90: 372-377, 2016.

9. Courcet JB, Elalaoui SC, Duplomb L, Tajir M, Rivière JB, Thevenon J, Gigot N, Marle N, Aral B, Duffourd Y, et al: Autosomal-recessive SASH1 variants associated with a new genodermatosis with pigmentation defects, palmoplantar keratoderma and skin carcinoma. Eur J Hum Genet 23: 957-962, 2015.

10. Martini M, Gnann A, Scheikl D, Holzmann B and Janssen KP: The candidate tumor suppressor SASH1 interacts with the actin cytoskeleton and stimulates cell-matrix adhesion. Int J Biochem Cell Biol 43: 1630-1640, 2011.

11. Kundumani-Sridharan V, Subramani J, Raghavan S, Maiti GP, Owens C, Walker T, Wasnick J, Idell S and Das KC: Short-duration hyperoxia causes genotoxicity in mouse lungs: Protection by volatile anesthetic isoflurane. Am J Physiol Lung Cell Mol Physiol 316: L903-L917, 2019.

12. Jiang Y, Xie X, Zhang Y, Luo X, Wang X, Fan F, Zheng D, Wang $Z$ and Chen Y: Regulation of G-protein signaling by RKTG via sequestration of the $G$ betagamma subunit to the Golgi apparatus. Mol Cell Biol 30: 78-90, 2010.

13. Englaro W, Rezzonico R, Durand-Clément M, Lallemand D, Ortonne JP and Ballotti R: Mitogen-activated protein kinase pathway and AP-1 are activated during cAMP-induced melanogenesis in B-16 melanoma cells. J Biol Chem 270: 24315-24320, 1995.

14. Bertolotto C, Abbe P, Hemesath TJ, Bille K, Fisher DE, Ortonne JP and Ballotti R: Microphthalmia gene product as a signal transducer in CAMP-induced differentiation of melanocytes. J Cell Biol 142: 827-835, 1998.

15. Hemesath TJ, Price ER, Takemoto C, Badalian T and Fisher DE: MAP kinase links the transcription factor Microphthalmia to c-Kit signalling in melanocytes. Nature 391: 298-301, 1998.

16. Primot A, Mogha A, Corre S, Roberts K, Debbache J, Adamski H, Dreno B, Khammari A, Lesimple T, Mereau A, et al: ERK-regulated differential expression of the Mitf $6 \mathrm{a} / \mathrm{b}$ splicing isoforms in melanoma. Pigment Cell Melanoma Res 23: 93-102, 2010.

17. Fock V, Gudmundsson SR, Gunnlaugsson HO, Stefansson JA, Ionasz V, Schepsky A, Viarigi J, Reynisson IE, Pogenberg V, Wilmanns M, et al: Subcellular localization and stability of MITF are modulated by the bHLH-Zip domain. Pigment Cell Melanoma Res 32: 41-54, 2019.

18. Bouché V, Espinosa AP, Leone L, Sardiello M, Ballabio A and Botas J: Drosophila Mitf regulates the V-ATPase and the lysosomal-autophagic pathway. Autophagy 12: 484-498, 2016.

19. Ichigawa $T$ and Hiraga $Y$ : A previously undescribed anomaly of pigmentation dyschromatosis universalis hereditaria. Jpn J Dermatol Urol 34: 360-364 1933 (In Japanese).

20. Zhang C, Li D, Zhang J, Chen X, Huang M, Archacki S, Tian Y, Ren W, Mei A, Zhang Q, et al: Mutations in ABCB6 cause dyschromatosis universalis hereditaria. J Invest Dermatol 133: 2221-2228, 2013.

21. Xie J, Zhang W, Zhang J, Lv QY and Luan YF: Downregulation of SASH1 correlates with poor prognosis in cervical cancer. Eur Rev Med Pharmacol Sci 21: 3781-3786, 2017.

22. Gong X, Wu J, Wu J, Liu J, Gu H and Shen H: Correlation of SASH1 expression and ultrasonographic features in breast cancer. Onco Targets Ther 10: 271-276, 2017. 
23. He P, Zhang HX, Sun CY, Chen CY and Jiang HQ: Overexpression of SASH1 inhibits the proliferation, invasion, and EMT in hepatocarcinoma cells. Oncol Res 24: 25-32, 2016.

24. Dubois F, Vandermoere F, Gernez A, Murphy J, Toth R, Chen S, Geraghty KM, Morrice NA and MacKintosh C: Differential 14-3-3 affinity capture reveals new downstream targets of phosphatidylinositol 3-kinase signaling. Mol Cell Proteomics 8: 2487-2499, 2009.

25. Coulombe P, Paliouras GN, Clayton A, Hussainkhel A, Fuller M, Jovanovic V, Dauphinee S, Umlandt P, Xiang P, Kyle AH, et al: Endothelial Sash1 is required for lung maturation through nitric oxide signaling. Cell Rep 27: 1769-1780.e4, 2019.

26. Price ER, Ding HF, Badalian T, Bhattacharya S, Takemoto C, Yao TP, Hemesath TJ and Fisher DE: Lineage-specific signaling in melanocytes. C-kit stimulation recruits p300/CBP to microphthalmia. J Biol Chem 273: 17983-17986, 1998.

27. Gonzalez GA and Montminy MR: Cyclic AMP stimulates somatostatin gene transcription by phosphorylation of CREB at serine 133. Cell 59: 675-680, 1989.

28. Jin ML, Park SY, Kim YH, Park G, Son HJ and Lee SJ: Suppression of $\alpha$-MSH and IBMX-induced melanogenesis by cordycepin via inhibition of CREB and MITF, and activation of PI3K/Akt and ERK-dependent mechanisms. Int J Mol Med 29: $119-124,2012$.
29. Kim HE, Ishihara A and Lee SG: The effects of Caffeoylserotonin on inhibition of melanogenesis through the downregulation of MITF via the reduction of intracellular cAMP and acceleration of ERK activation in B16 murine melanoma cells. BMB Rep 45: 724-729, 2012.

30. Saha B, Singh SK, Sarkar C, Bera R, Ratha J, Tobin DJ and Bhadra R: Activation of the Mitf promoter by lipid-stimulated activation of p38-stress signalling to CREB. Pigment Cell Res 19: 595-605, 2006.

31. Yun CY, You ST, Kim JH, Chung JH, Han SB, Shin EY and Kim EG: p21-activated kinase 4 critically regulates melanogenesis via activation of the CREB/MITF and $\beta$-catenin/MITF pathways. J Invest Dermatol 135: 1385-1394, 2015

32. Dauphinee SM, Clayton A, Hussainkhel A, Yang C, Park YJ, Fuller ME, Blonder J, Veenstra TD and Karsan A: SASH1 is a scaffold molecule in endothelial TLR4 signaling. J Immunol 191: 892-901, 2013.

This work is licensed under a Creative Commons Attribution-NonCommercial-NoDerivatives 4.0 International (CC BY-NC-ND 4.0) License. 